\title{
Mathematical Modeling of Enclosure Fires
}

Henri E. Mitler

US. DEPARTMENT OF COMMERCE National Institute of Standards and Technology

Building and Fire Research Laboratory

Gaithersbure, MD 20899

U.S. DEPARTMENT OF COMMERCE

Robert A. Mosbacher, Secrotary

MATIOMAL IASTITUTE OF STANDARDS

NND TECHNOLOGY

John W. Lyons, Director 



\title{
Mathematical Modeling of Enclosure Fires
}

\section{Henri E. Mitler}

\author{
U.S. DEPARTMENT OF COMMERCE \\ National Institute of Standards \\ and Technology \\ Building and Fire Research Laboratory \\ Gaithersbure, MD 20899
}

Issued May 1991

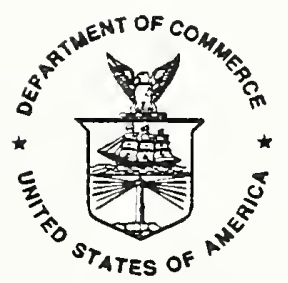

U.S. DEPARTMENT OF COMMERCE Robert A. Mosbacher, Secretary MATIONAL INSTIUTE OF STANDARDS AND TECHNOLOGY

John W. Lyons, Director 

List of Figures $\ldots \ldots \ldots \ldots \ldots \ldots \ldots \ldots \ldots \ldots \ldots \ldots \ldots \ldots \ldots \ldots \ldots \ldots$

Abstract $\ldots \ldots \ldots \ldots \ldots \ldots \ldots \ldots \ldots \ldots \ldots \ldots \ldots \ldots \ldots \ldots \ldots \ldots \ldots \ldots$

I. Preliminaries $\ldots \ldots \ldots \ldots \ldots \ldots \ldots \ldots \ldots \ldots \ldots \ldots \ldots \ldots \ldots \ldots$

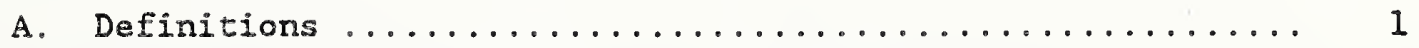

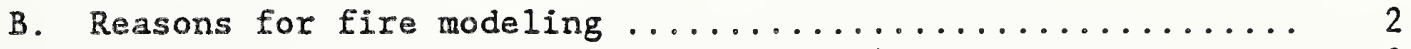

C. Short history of fire science and modeling $\ldots \ldots \ldots \ldots \ldots \ldots 2$

D. General remarks .......................... 3

II. The Physics of Fire $\ldots \ldots \ldots \ldots \ldots \ldots \ldots \ldots \ldots \ldots \ldots \ldots \ldots \ldots$

III. Field Models $\ldots \ldots \ldots \ldots \ldots \ldots \ldots \ldots \ldots \ldots \ldots \ldots \ldots \ldots \ldots \ldots \ldots$

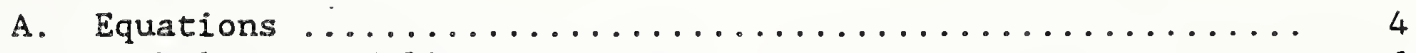

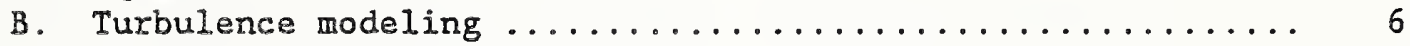

C. Solving the model equations $\ldots \ldots \ldots \ldots \ldots \ldots \ldots \ldots \ldots \ldots$

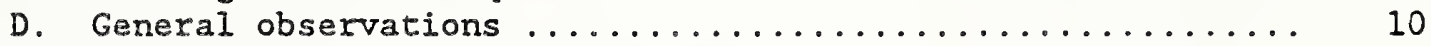

E. Solving the difference equations ................. 10

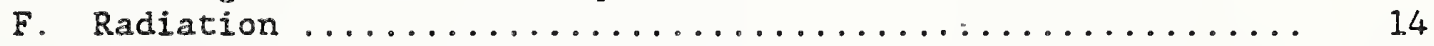

G. Calculations in three dimensions ................. 14

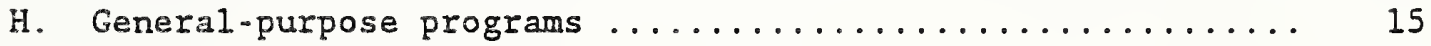

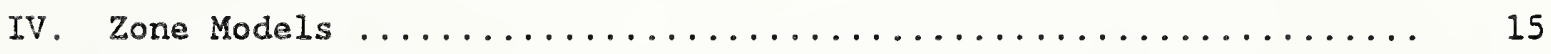

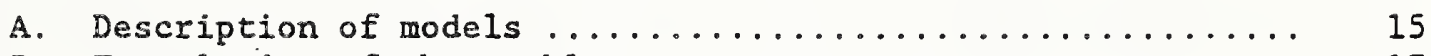

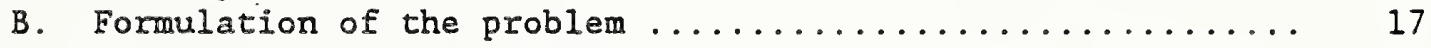

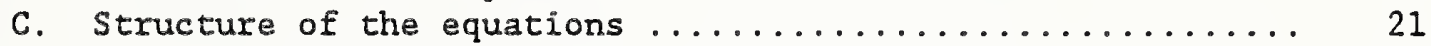

D. Numerical methods for solving the model equations ........ 22

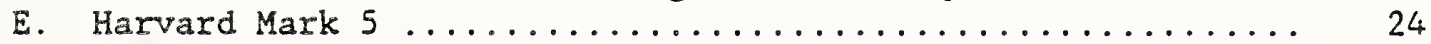

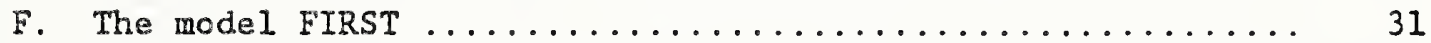

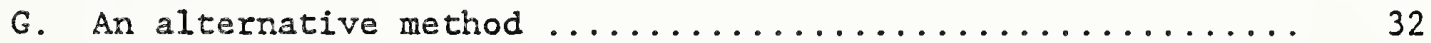

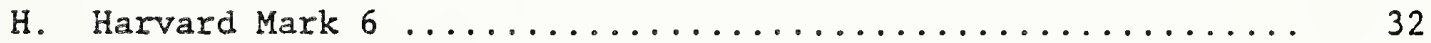

I. Validation ............................... 33

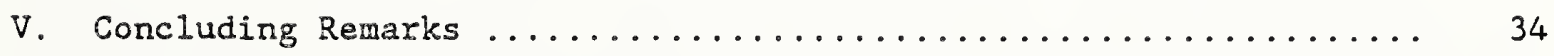

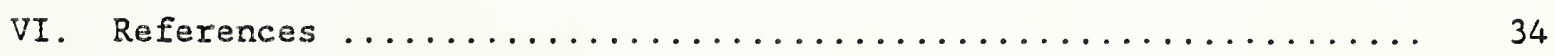




\section{LIST OF FIGURES}

Page

Figure 1. Schematic of the burning room, showing the various mass fluxes, $\dot{m}_{1}$, induced by a fire near the floor. The mean plume inclination is $\theta$, and the $Z_{1}^{\prime}$ 's are the heights of the interface, the soffit, the neutral plane, etc., above

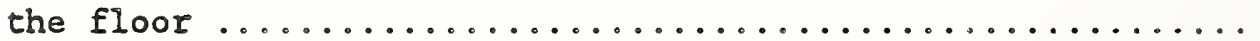




\title{
MATHEMATICAL MODELING OF ENCLOSURE FIRES
}

\author{
Henri E. Mitler \\ Building and Fire Research Laboratory \\ National Institute of Standards and Technology
}

\section{ABSTRACT}

After a brief description of the history and physics of enclosure fire modeling, two kinds of deterministic fire models are discussed: field models and zone models. The models consist of sets of coupled equations -. differential, algebraic, or a mixture of the two. Special emphasis is placed on discussing some of the numerical techniques used to solve these equations. Although this is not a comprehensive review article, an attempt has been made to give a sufficiently complete reference section that the interested reader can follow up on any item.

key words: computer models; fire models; mathematical models; modeling; numerical methods; room fires

\section{PRELTMINARIES}

\section{A. Definitions}

Pyrolysis is the heat decomposition of large molecules, generally in a solid, into smaller molecules; these exit the solid in the form of gas or vapor. Pyrolysis is often loosely referred to as burning. A fire is a set of physical and chemical phenomena which include combustion (the rapid, localized exothermic chemical reactions involving an oxidizer, almost always atmospheric oxygen, which produce high temperatures and luminosity), fluid flows, and (generally) pyrolysis or evaporation. If the combustion is in the solid phase, as in char oxidation, it is a smoldering fire. When the combustion occurs in the gas phase, the luminous part of the gas is called the flame. Thus, fire is the more general phenomenon, that may or may not involve a flane. Enclosure fire models attempt to describe all of the important phenomena associated with a fire in an enclosure, such as the production of various species, fluid flow throughout a building, and structural damage due to heat. Flame models attempt to describe the structure, properties, and behavior of the gas-phase combustion zone only (see Jeng et al. (1982), Fernandez-Pello (1984), Sibulkin (1988)).

When a fire in an enclosure grows large enough, a number of phenomena frequently ensue: the temperature of the hot gases in the room rises with increasing speed to $600^{\circ} \mathrm{C}$ or more; the accompanying heat fluxes to the floor rise to $2 \mathrm{~W} / \mathrm{cm}^{2}$ or more, and other objects ignite. By the time a second object ignites, it is usually only a matter of seconds before most of the flammable materials in the room burst into flame. The smokey layer descends nearly to the floor, and often flames shoot out of the enclosure. All or most of these events take place over a period of a few seconds; this sequence of events is called flashover. After flashover, the room is said to be "fully involved." 
This paper discusses deterministic models of enclosure fires, which are a subset of fire models. Thus we have not included stochastic models of room fires (Siu, 1981), forest fires (Rothermel, 1972), hybrid models (which contain features of two or more distinct models), or smoldering fires, which range from cigarette fires to fires in mines (Ohlemiller 1985; Mitler 1988). The emphasis will be on numerical methods.

\section{B. Reasons for fire modeling}

It is desirable to have a good mathematical model for the development of a fire in a structure in order to:

1. Avoid full-scale testing. For example, new materials and new or altered suppression devices all need to be tested before they can be used in new construction. Once the intrinsic properties of the materials are known, it is much faster and cheaper to estimate the performance of such new materials and designs on a computer than to carry out full-scale tests. Moreover, the fire behavior of a new furniture item, for example, could be tested in a number of configurations rather than just the one that would be tried in a physical test. Similarly, the behavior of a fire when the venting is altered, when the wall thickness is changed, or any combination of structural changes are made, can be examined in a way which would otherwise be impossible.

2: Help designers and architects. The most important parameters determining the course of any particular fire can be found by making a number of runs, and so details of design can be optimized according to a desired criterion.

3. Establish the flammability of materials. There is as yet no universally accepted definition of "flammability." Indeed, the fire behavior of any material is not an inherent property of it, but depends on the circumstances and configuration in which the material is burning, as well. A reliable model permits the assessment of material flammability in a particular configuration.

4. Increase the flexibility and reliability of fire codes. At present, fire codes generally prescribe rigid specifications. With models, performance fire codes become possible.

5. Identify needed fire research. The construction and use of such models very quickly reveal where there are significant gaps in our knowledge, and indicate where research efforts should be focused.

6. Help in fire investigations and litigation. A good model will be very useful in the investigation and reconstruction of fires, whether for litigation or for other reasons. Indeed, some models have already been so used.

\section{Short history of fire science and model:ng}

Mathematical modeling of enclosure fires dates back at least sixty years, if we take Ingberg's (1927) research at the National Bureau of Standards as the first attempt to understand scientifically the post-flashover compartment fire. Ingberg examined the fire behavior of a fully-involved room, burning in 
a quasi-steady state. He related the temperature history of the room gases to the available mass of fuel.

It took thirty years before that analysis was improved: Kawagoe (1958) found that the burning rate was limited to a factor times $\mathrm{A} / \mathrm{H}$ (the "ventilation factor"), where $A$ is the area of the ventilating opening and $H$ its height. Kawagoe's theoretical results for a steady-state fire in a flashedover room where the gases are well mixed were largely confirmed by experiment (Thomas 1960; Simms et al. 1960; Gross and Robertson 1965). Later, these results and analyses were extended by Odeen (1963). Thomas and Hinkley (1964), and Thomas et al. (1967) introduced the idea that the hot gases form a separate upper layer, and that the thermal plume is the way in which mass and energy pass into the upper layer.

Interest in fire research and in fire modeling grew rapidly after 1958, and in 1959 the first symposium on fire research and the use of models was held in Washington, sponsored by the National Academy of Sciences (Berl, 1961). Part of the reason for the enhanced interest was that research on the physics and chemistry of fires had made significant strides. Equally important was that the development of large, fast electronic computers permitted the rapid solution of many simultaneous coupled equations, and therefore the possibility of more sophisticated and comprehensive mathematical models. Research since then has been accelerating, as has the development of computer hardware and appropriate mathematical software.

\section{General renarks}

Mathematical models can be put into two broad classes: (a) stochastic (or probabilistic), and (b) deterministic. In stochastic models, the probabilities and contingent probabilities of significant events are estimated, and then the a priori probability of a particular outcome estimated. Little or no physics needs to be introduced (Thomas, 1967; Williamson, 1976; Siu, 1981; Schmidt, 1979).

In deterministic models, the problem and the configuration are prescribed, and then the laws of physics and chemistry, as reflected in the equations chosen to simulate reality, are invoked. Well-deternined correlations may be used to describe some of the processes. These equations then determine the evolution of the fire. No probabilities are involved.

There are by now many exemplars of each kind of model. We shall concentrate on deterministic models. The review will not be comprehensive: only a small subset of existing models will be described. The numerical techniques used in these models, however, are fairly representative of those in general use.

Finally, it should be made clear that the adequacy with which the physics and chemistry of the processes taking place in fire is represented by the model determines the adequacy of the results. That is, it is far more important than the precision of the numerical solutions; thus, integrating the equations to within 18 (or even 58 ) is perfectly adequate. 


\section{THE PHYSICS OF FIRE}

There are a number of discussions of the physics of fires (for example, Mitler 1978, Lyons et al. 1980, Drysdale 1985, Lyons 1985, DiNenno et al. 1988). Since the emphasis in this book is on numerical methods, I shall here sketch the rudiments of only the most important dynamical processes.

A fire acts as a pump, since the hot gases produced by combustion expand, rise because of buoyancy, form a thermal plume, and produce large-scale convection. This plume contains products of combustion, including $C O$ and soot (besides $\mathrm{CO}$, other common toxicants are $\mathrm{HCl}$ and $\mathrm{HCN}$ ), and is oxygen-vitiated. There are other associated flows, such as entrainment of air into the thermal plume. The plume, upon striking the ceiling, fans out into a ceiling jet; this rapidly flowing layer eventually reaches the confining walls, and is turned downward, forming "wall jets." Momentum carries these down the walls for some distance, but eventually that is dissipated and buoyancy turns them back up. Then they form a hot gas layer trapped under the ceiling; this layer heats up the ceiling and the contiguous upper parts of the walls. It is sooty and oxygen-poor (i.e., lean in oxygen), since the plume is. Initially, expansion drives cool air from the room. When the layer becomes so thick that its lower boundary is driven below the top sill of a free vent, the hot gases begin to escape from the compartment, too. After a brief phase during which room gases -- hot and cold --continue to escape over the entire vent(s) due to the gas expansion, the buoyant outflow reaches such a magnitude that external (generally cooler) air is pulled into the room. In the region between the two gas streams, a mixing region is formed, so that some of the hot gases are pulled back into the room and mixed with the lower layer. The situation is shown schematically in Fig.1, taken from Steckler et al.(1982).

The flows are generally turbulent due to two mechanisms: first, as a gas mass accelerates due to buoyancy, it will quickly achieve velocities such that the flow becomes unstable. Second, gradients in the flow velocity will produce vortices, which eventually dominate the plume.

The flames produce radiation which heats walls and other objects in the enclosure. The heat feedback from other hot regions such as the hot layer and the ceiling will also contribute, and exposed objects may be heated to their ignition temperature. Moreover, the additional heat feedback will generally accelerate the spread and burning rates of the fire(s).

Thus, many processes take place in a fire, and must be described in a good model. Among these are convective heat and mass transfer, radiative heat transfer, ignition, pyrolysis, and the formation of soot and other species.

\section{FIELD MODELS}

\section{A. Equaticns}

Field models attempt to calculate the velocity and temperature fields in an enclosure, given some (generally prescribed) heat source. Some field models also calculate gas species concentrations as a function of position and time. The equations which describe the flow of fluids are the conservation of mass, momentum, and energy, plus the equation of state of the fluid. These equations constitute a set of coupled nonlinear partial differential equations (PDEs). 
The associated boundary conditions and initial conditions are determined by the particular problem and its geometry (see, for example, Batchelor, 1967).

Conservation of mass is described by the continuity equation

$$
\partial \rho / \partial t+\nabla \cdot(\rho \mathbf{u})=S \text {. }
$$

Here, $\rho$ is the local mass density of the fluid and $\mathbf{u}$ is its velocity. The $S$ on the right-hand side corresponds to sources or sinks of mass; for almost all cases of interest, $S$ is zero. The Navier-Stokes equation describes conservation of momentum:

$$
\partial(\rho \mathbf{u}) / \partial t+\nabla \cdot(\rho \mathbf{u u})=-\nabla \mathrm{p}-\mathrm{g} \rho \mathbf{e}_{3}+\nabla \cdot \boldsymbol{\tau},
$$

where $g$ is the acceleration of gravity, $p$ is the absolute pressure, $e_{3}$ is the upward-pointing unit vector, and $r$ is the stress tensor. The right-hand side describes the forces acting on the differential element.

Because the kinetic energy and the viscous dissipation terms are generally small compared to the internal energy, they are usually neglected. The pressure work is included in the PV term in the enthalpy. Hence the energy equation is approximated by

$$
\partial\left(\rho c_{p} T\right) / \partial t+\nabla \cdot\left(\rho c_{p} T u\right)=\dot{q}^{\prime \prime \prime}-\nabla \cdot q,
$$

where the right-hand side gives the sources:

$$
\dot{q}^{\prime \prime \prime}=\text { volumetric source, } \quad \mathbf{q} \equiv \text { heat flux }=-k \nabla T \text {. }
$$

The viscosity $\mu$ implicit in Eq. (2) and the thermal conductivity $k$ which is implicit in Eq. (3a) via Eq. (3b), are functions of $\rho$ and $\mathrm{T}$. Finally, there must be an equation of state,

$$
\rho=\rho(p, T) \text {, }
$$

for which the ideal gas law is generally used:

$$
\mathrm{p}=\rho \mathrm{RT} \text {. }
$$

Analytic solutions of these equations exist for special cases that have simple boundary conditions. Thus in the steady state, the momentum equations are elliptic, and admit of analytic solutions for the two-dimensional case. In the general two-dimensional case, a stream-function formulation that simplifies the equations becomes possible, although they still remain nonlinear. The assumption of no viscosity also simplifies the problem a great deal, as does the assumption that the fluid is incompressible, in the sense that $\partial \rho / \partial \mathrm{p}=0$. In other special cases, simplifications become possible which either permit analytic solutions to be found or make the task of solving the numerical equations easier. However, the general set of equations is not analytically solvable. Therefore, solving the equations usually requires the use of numerical techniques (Launder and Spalding, 1972; Roache, 1976; Patankar, 1980; Markatos and Cox, 1984).

In order to solve the PDE's numerically, the equations must be discretized; that is, the region is divided, by a set of grids, into as many small volume elements as is practicable, and the PDE's are replaced by a set of difference equations (see, for example, Atkinson, 1978). 
We will discuss the equations and the solution methods in more detail, in the rest of Section III. First, consider the approximations made to the basic equations. Since the equations are parabolic (elliptic in the steady state), the boundary conditions must be specified over the entire boundary of the computation region. The no-slip condition for the velocity components on solid boundaries is used ( $\mathrm{Ku}$ et al. 1977) and either the temperature, heat flux, or a combination of the two is prescribed for the thermal boundary condition. At an open boundary such as a doorway or window, the free flow of gas into and/or out from the computational region must be permitted, and the flow field in the outside region must be known or postulated.

The equations are sometimes simplified by assuming the fluid to be incompressible. Moreover, the variation of other fluid properties, such as viscosity, specific heat, and thermal conductivity, is small compared to that of the corresponding eddy properties and is therefore often neglected. Fusegi and Farouk (1988) allow full compressibility and variable properties, with viscous flow. They use a $\mathrm{k}-\epsilon$ turbulence model (see below). Also see Galea (1989), Larson and Viskanta (1986), Patankar (1974), and Yang et al. (1984).

The source of convective and radiative energy is the chemical release from combustion. This is generally specified as an input to the program. Bagnaro et al. (1981), on the other hand, modeled the combustion rate by finding a diffusion equation for the mixture fraction; this also provides species concentrations.

\section{B. Turbulence modeling}

The flows generated in room fires generally are turbulent, so that they consist of eddies or vortices of many sizes. The energy which resides in large vortices cascades down to smaller and smaller vortices, until it diffuses into heat. The size of the turbulent eddies that are generated by the numerical approximations to the solutions of the flow equations can be no smaller than at least several computational cells; the actual eddies exist down to the size where the viscous forces dominate over inertial forces and energy is dissipated into heat. This is commonly referred to as the Kolmogoroff microscale. For laboratory-sized turbulent fires, this scale is of the order of a millimeter, which is much smaller than the usual computational mesh size. Since the mesh spacing is usually much greater than the Kolmogoroff microscale, special methods must be used to approximate the turbulence in these numerical approaches. Thus, the turbulence is often modeled by turbulent transport equations which are then coupled to the full set of equations.

When the turbulent fluctuations are very rapid, the instantaneous solutions are difficult if not impossible to obtain. Indeed, turbulent fluctuations generally exist even in what would macroscopically be described as a steady state. Therefore, one often solves for the time-averaged, or Reynoldsaveraged, flows. A variable $\phi$ is written as

$$
\phi=\hat{\phi}+\phi^{\prime}
$$

where $\hat{\phi}$ is the time-averaged value and $\phi^{\prime}$ is the fluctuating component. When this is done, there are more variables than equations, and the Reynolds stress terms (defined below) must be given separately in order to achieve closure. One way frequently used to handle those terms in turbulent flow is by the 
Spalding $k-\epsilon$ model of hydrodynamic turbulence. This turbulence model is due to Saffman (1970), Rodi and Spalding (1970), Jones and Launder (1972), and Launder and Spalding (1972). The generic name for this type of correction to the basic equations is subgrid modeling.

The $k-\epsilon$ model is obtained as follows: Each variable is expressed as a time-averaged value plus a fluctuating component, as in Eq.(6). However, it is generally assumed that density fluctuations $\rho^{\prime}$ are sufficiently small to justify neglecting turbulent correlations involving then (the Boussinesq approximation, which assumes that the flow has constant density except in the buoyancy term). These variables are substituted into the flow equations (1) and (2), then time-averaged. The resulting equations are

$$
\operatorname{div} \hat{\rho} \hat{\mathbf{u}}=0
$$

and

$$
\hat{\rho} \partial \hat{\mathbf{u}} / \partial t+\hat{\rho} \hat{\mathbf{u}} \cdot \nabla \hat{\mathbf{u}}=-\nabla \hat{\mathrm{p}}+\nabla \cdot(\mu \nabla \hat{\mathbf{u}})-\hat{\rho} \partial \mathrm{R}_{1 \mathrm{j}} / \partial \mathrm{x}_{\mathrm{j}}
$$

where the sumation convention has been used in the last term, and the tensor $R$ is the Reynolds stress (per unit mass):

$$
R_{1 j}=\left\langle u_{i}^{\prime} u_{j}^{\prime}\right\rangle
$$

where the brackets indicate time averaging. The $k-\epsilon$ model arises from the determination of the form of this correlation term. One approach, which is far simpler than using the full Reynolds stress model, is to use an algebraic expression. Thus, the Boussinesq turbulence, or eddy viscosity, $\mu_{t}$, which accounts for the momentum transfer due to turbulent fluctuations, can be used by choosing

$$
-\hat{\rho} \Sigma \partial R_{1 j} / \partial x_{j}=\mu_{t} \nabla^{2} \hat{u}_{1} \text {. }
$$

Prandtl suggested that, in analogy with $\mu$ in the kinetic theory of gases,

$$
\mu_{\mathrm{t}} \propto \rho u_{\mathrm{t}} \mathrm{L}_{\mathrm{t}}
$$

where $u_{t}$ is a local turbulent velocity and $L_{t}$ is a characteristic turbulent length scale. If one can find simple algebraic expressions for $u_{t}$ and $L_{t}$, then the problem formulation is complete. It is not difficult to show that

$$
\mu_{t}=C_{1} \hat{\rho} k^{2} / \epsilon
$$

where

$$
k \equiv\left\langle u_{1}^{\prime} u_{1}^{\prime}\right\rangle / 2
$$

is the mean turbulent kinetic energy per unit mass, $\epsilon$ is the rate at which $k$ is dissipated, and $C_{1}$ is a constant to be determined. Since two new variables $k$ and $\epsilon$ have been introduced, it is necessary to solve two new transport equations. These so-called two-equation models have been described by Saffman, by Spalding, and by Launder. Also see Peyret and Taylor (1983), Chapter 9.

The $k-\epsilon$ model ignores the effects of gravity, yet it is the gravitational force which gives rise to buoyancy, which in turn accelerates flows and can give rise to turbulence. When buoyancy is important, the anisotropy forces 
the determination of the level of temperature fluctuations. This quantity is symbolized by $g$ :

$$
g=\left\langle T^{\prime 2}\right\rangle
$$

where $T^{\prime}$ is the fluctuating component of $T$, and a third transport equation is needed. This buoyancy-corrected $k-\epsilon$ model should be used whenever large-scale recirculating movements occur, such as in a fire. See Markatos et al. (1982). The $k-\epsilon=g$ model is described in Launder and Spalding (1972).

The transport equations for turbulent quantities are usually treated by the $k-\epsilon$ model, but with no viscous terms, since the molecular viscosity is so much smaller than the turbulent viscosity (see, e.g., Fusegi and Farouk, 1988). For turbulence, $\mathrm{Ku}$ et al. (1977) used the algebraic turbulence viscosity model of Launder and Spalding (1972):

$$
\begin{aligned}
& r_{x x}=2 \mu_{\theta} \partial u / \partial x \\
& r_{x y}=\mu_{\theta}(\partial u / \partial y+\partial v / \partial x) \\
& q_{x}=-k_{\theta} \partial r / \partial x \\
& q_{y}=-k_{\theta} \partial r / \partial y
\end{aligned}
$$

where $\mu_{0}$ and $k_{e}$ are effective values of viscosity and thermal conductivity. The turbulent flow formulation is sometimes Favre averaged, which means that new variables are defined as integrals with $\rho$ as the weight function. This is used to describe flows with large density variations (Fusegi, 1988).

As Baum and Rehm (1984) point out, most turbulence models in use are of the gradient diffusion type. Thus, they implicitly assume that a small-scale, locally homogeneous turbulent field underlies the organized macroscopic mean motion whose solution is sought. There is evidence that this is not correct; for that reason, among others, Baum and Rehm do not use turbulence models in their work. They aver that, on the contrary, the large eddies control the overall fluid motion, and therefore theirs is a large-eddy model. Since the energy cascades down from large to small eddies, the kinetic energy would, with their model, accumulate (unphysically) at the grid scale, rather than diffusing into heat. Therefore they periodically use Lanczos smoothing (which is equivalent to putting in an artificial viscosity term. See Rehm et al., 1982). A detailed discussion of their model is given in section III.E. 2.

\section{Solving the model equations}

The three principal means of discretizing the equations are the use of finite differences, finite elements, and finite volumes. In using finite differences, the differential operators are approximated by making a Taylorseries expansion of the operators in terms of differences on a grid, and taking a finite subset. These are described in Chap. 2 of Peyret and Taylor (1983). The finite-element method basically consists of reducing the PDE's to a set of ODE's or algebraic equations; one typically assumes an expansion of the function in terms of a basis. This could include polynomial fitting. See Chap. 3 of Peyret and Taylor (1983). Finally, the finite-volume method involves integrating the governing equations along the faces of control volumes surrounding each node. This has the advantage of satisfying the conservation 
laws in the difference equation form. This is particularly easy to do for equations (1)-(3), for they are written in what is called divergence, or conservation-law, form; these labels are used because in integrating over a volume, the divergence theorem can be used on the second terms on the lefthand side to transform them into the fluxes of each quantity across the enclosing surface. When the volume is that of a primitive cell, this guarantees that the flux leaving one cell flows into the adjacent one, conserving the flux, both in the small and globally. This leads to a set of algebraic equations involving scalar terms evaluated at the center of the cell and flux terms at the cell boundaries. Thus the PDE's describing fluid flow are approximated by a (large) set of algebraic equations. That is true, of course, whatever discretization method is used.

Birkhoff (1983) has written an excellent review article which places the numerical solutions of these equations in perspective (see, especially, his section 13). A classic text on PDE's and their numerical solution is that by Richtmyer and Morton (1967); a more up-to-date text is that by Peyret and Taylor (1983).

The method most generally used for the solution of the nonlinear difference equations is some variant of the Newton-Raphson multivariate procedure (see section IV.E.1); this is equally useful for sets of nonlinear equations. This technique generally requires the evaluation of the Jacobian of the equations.

For the steady-state case, the Navier-Stokes equations are elliptic (in the spatial vaxiables). If the problem is one-dimensional and there is no radiation, the Jacobian is tridiagonal, and the solution method is easy (Richtmyer and Morton, 1967). The non-linear Gauss-Seidel method (see sections III.E.1 and IV.E) can also be used, but is not as good as Newton's method for this case. For the two-dimensional case, there are five diagonals and seven diagonals in three dimensions, but the matrix is still sparse, and special methods can be used to find the solution. The best way to solve them is by iterative techniques, rather than "exactly," because truncation errors can build up to prohibitive levels. When radiation is included in the problem, every cell connects with every other (that is, there are no zero matrix elements), and no special solution methods are available.

The prototypjcal problem is a prescribed fire in a room with one or more vents. Explicit methods have the theoretical advantage of yielding exact answers in one pass; however, they may require exceedingly short time steps. Implicit methods are more stable, and permit much larger time steps, usually more than making up for the fact that iterations are necessary for convergence to a solution. An early comparison of explicit vs implicit methods was made by Torrance (1968).

Because of computer memory and speed constraints, the equations have generally been written assuming two-dimensional flow. For example, Ku et al. (1977) wrote the program UNDSAFE to solve the Navier-Stokes equations with local heating, in two dimensions. They solved the problem for rectangular enclosures. They used the SIMPLE algorithm of Patankar and Spalding (1972). The governing equations and their boundary conditions are approximated with finite-difference equations by a control-volune-based method. The computing cells are chosen so that their boundaries coincide with physical boundaries. A staggered grid system originally devised by Harlow and Welch (see Harlow and Amsden, 1971) was chosen: in this system the pressure, temperature, and density are evaluated at the centers of the cells while the velocity com- 
ponents are evaluated at the cell boundaries. Thus the momentum equations are written for cells centered at the boundaries of the basic cells. The convection-diffusion terms are discretized by a hybrid scheme (Patankar, 1980, pp.89-90 and 126-131).

\section{General observations}

Numerical approximations lead to approximate solutions; sometimes the solutions are then so poor that the results are incorrect, but that might not be apparent, and would be attributed to the physics of the problem. For example, numerical diffusion or dispersion (or both) are created by the discretization. Thus, fine structure is smeared out (or ripples created) via the continuity equation; an artificial viscosity is created in the momentum equation, and numerical conductivity in the energy equation. The following is one example: the limitations of computer speed and size forced Torrance and Rockett (1969) to use a relatively gross grid. The solution of the equations showed that at high Grashof numbers ( $\mathrm{Gr}$ - 1011) large coherent structures were periodically formed and shed, and eventually dissipated. It was surmised that this resulted from the large shear produced across the large mesh (because of the coherence of the phenomenon, they avoided saying that this was the onset of turbulence). They also found that their numerical solution of the equations resulted in unphysical oscillations. They therefore devised a smoothing algorithm which eliminated these oscillations; note that this smoothing is equivalent to the addition of a zeroth-order viscous term to the equations. In fact, later reconsideration of the same problem using a smaller grid did not produce these eddies, showing that they were an artifact of the (too-large) grid size: the numerical viscosity was much reduced when the grid size was made smaller.

This example and others lead to an important general guideline: it is essential to make sure that the numerical procedure yields reasonable solutions. This is perhaps best done by showing that the numerical procedure used yields the correct solution for a case where an analytic solution is known (Birkhoff, 1983). For example, Torrance (1968) could not obtain an analytic solution to the fluid-flow equations for a problem in which the Grashof number was greater than $10^{5}$, so that a comparison was not possible. However, the analytic solution for the problem with $\mathrm{Gr} \simeq 0$ was available, and the validity of the solution in that limit, at least, was verified.

Again, Baum (1976) examined the $k, \epsilon, g$ model as follows: he found quasianalytic solutions for two boundary-value problems of flow past a plate. He then solved the same problems using the $k, \epsilon, g$ model, and showed that the parameter values normally used for the model lead to some internal singularities. To obtain numerical solutions, one integrates across these, and the result depends on the relative location of the nodes and the singularities. Thus solutions obtained with the $k, \epsilon, g$ model are not always well-defined.

\section{E. Solving the difference equations}

Once the equations have been discretized, they must then be solved. Many ways have been used to solve these sets of simultaneous algebraic equations. 


\section{Linear methods}

Since the Navier-Stokes equations are nonlinear, the resulting difference equations are also nonlinear. One solution method is to solve a sequence of sets of linear equations, although this is seldom the most accurate way. Therefore we first consider solution methods for sets of linear equations; that is, sets which can be written in the form

$$
\mathbf{A x}-\mathbf{b},
$$

where $\mathbf{A}$ is a given matrix, $\mathbf{b}$ a given vector, and $\mathbf{x}$ the vector we are trying to find. An enormous literature exists on the solution of this problem, and we shall not attempt to describe all the methods. A possible explicit method is to find the inverse of the matrix $\mathbf{A}$, and to set

$$
\mathbf{x}=\mathbf{A}^{-1} \mathbf{b}
$$

This technique has many drawbacks, however, and is therefore almost never used; the problem is usually attacked in the form (19). Solving for $\mathbf{x}$ generally involves Gaussian elimination or some variant of it. Even when the matrix $\mathbf{A}$ is nonsingular, however, the procedure is inadequate when its condition number $c(A)$ (Ralston, 1965) is so large that the product of the relative exror in the matrix (due to rounding or truncation errors in the calculation) and $c(A)$ is of order one or larger. This is made more precise by Eq.(9.4-20) in Ralston, for example.

Besides explicit methods, there are iterative procedures, such as the

Jacobi or Gauss-Seidel (hereafter referred to as G-S) successive substitution methods (we shall describe these later). The G-S proceduxe is one of a class of relaxation procedures, where one or more components of the residual vector

$$
\boldsymbol{r}_{1}=\mathrm{b}-\mathrm{Ax}_{\mathrm{i}}
$$

arising in the ith iteration is (are) made to vanish by some modification ("relaxation") of the ith approximation $x_{i}$. These methods are especially important, because they also are useful when the equations are nonlinear.

There are a number of iterative techniques that are based on minimizing a quadratic form. Two of these are the method of steepest descents and the conjugate gradient method. For ill-conditioned systems, the convergence rate of steepest descents will be very slow; it is mentioned here, because it appears in the Levenburg-Marquard method, discussed in Section IV.G. The conjugate-gradient method (Ralston, 1965, section 9.8-3) will converge in a finite number of steps, but that number may be large, and it is often not as efficient as the relaxation methods. This is not always the case, however: see Lewis and Rehm (1980).

\section{Current methods}

An implicit iterative method is generally used to solve the equations. I cannot do better than to quote $\mathrm{Ku}$ et al. (1977), who solved the flow problem in a compartment with a fixe which was modeled as a prescribed heat source, to describe the details of the numerical procedure: "The flux terms at the cell boundaries are approximated through upwind differencing* while the velocity

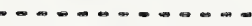

* Author's note: for discussions of upwind differencing, see Raithby and Torrance (1974); Peyret and Taylor (1983), section 2.4; Drummond (1989) 
and temperature gradients on the cell boundaries are approximated by central differences. The unsteady term is approximated by a simple forward difference in time. The overall procedure is conservative in that it conserves mass, momentum, and energy in any finite region of computation."

"After the above procedures are carried out one obtains, at each cell, five algebraic equations in five unknowns: pressure, density, temperature, and the two velocity components. In low speed flows the pressure is only weakly coupled to the equation of state* and thus the primary coupling in the state equation is between the density and temperature. Therefore the usual procedures employing the equation of state to calculate the pressure cannot be used. Special procedures must be devised for treating the pressure."

The special procedure they use to find the pressure is an iterative scheme, following Patankar and Spalding (1972): "the temperature is evaluated from the energy equation. Then the density is found from the equation of state. This reflects the correct physical mechanism that density changes are caused primarily by temperature changes. Next a pressure field is estimated by taking it to be the pressure field at the previous time level. Using this first estimate of the pressure field the velocity components are calculated from the two momentum equations. These velocity components, in general, do not satisfy the mass conservation equation so there will by a residual mass source at each cell. The pressure is then corrected in such a manner that the residual mass sources are reduced in size. This corrected pressure is then used as a second estimate to calculate the velocity components. The process is repeated through a number of iterations until the residual mass sources become as small as desired. Thus the final pressure and velocity fields will satisfy the mass conservation equation with a prescribed accuracy at each cell. The equation for the pressure correction is obtained by making the approximation that the velocity corrections are linearly dependent on the gradient of the pressure correction." $\mathrm{Ku}$ et al. go on to describe the algorithm in further detail.

The two-dimensional steady-state problem was solved in a similar way by Markatos and Cox (1981). The solution method was a finite-difference technique which combines features of the SIMPLE and NEAT algorithms (Patankar and Spalding (1972) and Spalding (1977), p.565) together with a whole-field pressure-correction algorithm described by Markatos et al. (1982).

A slight modification was used by Fusegi and Farouk (1988): they also use an iterative scheme (a modified SIMPLE algorithm). However, their solution method employs the Strongly Implicit Scheme (Stone, 1968) as a multidimensional simultaneous algebraic equation solver instead of the less efficient Iine-by-line solver based on the tridiagonal matrix algorithm used in the standard method (Patankar, 1980). They solve the equations using a pressurecorrected iterative technique.

In the late $70^{\prime} \mathrm{s}$, Baum and Rehm launched a project to model the combustion and convective flow processes which occur in enclosure fires, from first principles. The first paper (Rehm and Baum, 1978) established the equations to be solved, taking the following conditions: the fluid is assumed to be inviscid, thermal conductivity is ignored, the gas is ideal, the enclosure walls are perfectly insulating (so that all the processes are adiabatic), and -......................

* Author's note: that is, the pressure during well-vented fires remains approximately atmospheric. 
the heat source, which simulates a fire and drives the flow, is prescribed (both in time and in extent). The enclosure is taken to be two dimensional, with a rectangular cross-section. Radiation transport was ignored. Unlike many such studies, however, the Boussinesq approximation (approximately constant density) was not made. That is, the large density variations due to the equally large temperature variations are taken into account, but compressibility effects were suppressed. Such a fluid has been called "thermally expandable" (Porsching, 1977). The flow is fully time-dependent, except that no turbulence model is included, and acoustic oscillations are not in the equations. The latter result is achieved by treating the pressure terms so that only the time derivative of the spatially averaged pressure appears in the energy equation, but the spatial gradients of the pressure are admitted in the momentum equation. In that way, buoyant or internal-wave motions can appear, but sound waves are "filtered out."

These equations were solved in the second paper of the series (Baum et al. 1982). They did not use an available "package;" rather, they constructed their own scheme. First, the equations were nondimensionalized in such a way that all dependent quantities were made of order unity, which is one way to avoid certain computational inaccuracies (see section IV.E.3). Next, the equations were discretized; they established a number of criteria which the differencing scheme must satisfy. By casting the equations in conservationlaw form, the divergence and the curl (for the vorticity equation) are correctly calculated when in discrete form. Second, the difference equations must be at least second-order accurate in time as well as space. This was done by using a three-time-level, leap-frog scheme to express time derivatives numexically, rather than the usual two-level central difference formula. Third, they should accurately reproduce internal-wave modes; in Baum and Rehm (1984) they had already established which difference equations satisfy this critexion. They also carefully avoided using differencing schemes which had been shown to lead to computational instabilities. The scheme they chose is called $\mathrm{J}_{3}$ by Arakawa and Lamb (1977). They also were able to avoid an instability described by Kreis and Oliger (1973), by using a different order in the operations.

They use a typical staggered grid (Harlow and Amsden, 1971). The pressure (energy) equation plus boundary conditions constitute a singular linear algebraic system of equations; details of the algorithm used to solve them are given in Lewis and Rehm (1980). They also carried out a linear stability analysis, and found a condition on the maximum time-step size that can be taken at any step. In the formulation of their field model, Baum et al (1983) carefully and explicitly checked the adequacy of their numerical solution against the exact analytic solutions for some cases where the latter could be found; the importance of this step was stressed in Section III.A.

More discussion of the numerical procedure is given in Baum and Rehm (1982). In that report they also give some experimental verification of the results for the formation and buoyant rise of a thermal plume due to a heat source in an enclosure. In Part II (Rehm et al., 1985) they carry out the task which is usually omitted in these numerical calculations: they verify the adequacy of the algorithm. They find exact analytic solutions to the equations for two special cases, and find that the numerically-derived solutions agree with those to about one part in $10^{6}$ (the specified accuracy) in both cases. In Baum et a1. (1982), the computation was extended to treat the coagulation of the smoke aerosol produced by the fire. Baum and Rehm (1984a) extended the computation to the three-dimensional case. In their 
words, "the basic assumption is that large-scale macroscopically resolvable motion is responsible for most transport of mass, momentum, and energy except at boundaries. Small-scale physical and chemical processes, which control heat and mass transfer to boundaries, as well as most combustion and smoke aerosol phenomena, are ultimately to be embedded in a systematic way into the large-scale flow." As in the first calculations, heat transfer to the boundaries is ignored, and no turbulence model is employed. The large-scale transport is then calculated directly from the equations of motion.

\section{F. Radiation}

There has been much less uniformity of treatment for radiation than for fluid flow. In fire models, radiation has either been omitted altogether (Ku et al. 1977, Markatos et al. 1982), or questionable assumptions have been made: radiation has been assumed not to interact with the gas (Handa et al. 1984), the gas is assumed to be gray (Bagnaro et al. 1983, Markatos and Pericleus 1983), or the radiation field has been considered to be one-dimensional (Lloyd et al. 1979).

The interaction between turbulent convection and radiation in room fires has recently been investigated by Fusegi and Farouk (1988) in a two-dimensional calculation. Their calculation is typical: the number of nodes they use is about 20,000. Since they use an implicit technique for the solution, they must iterate: 50-100 iterations are needed per time step (but they are giant time steps: $\Delta t$ - 10 to $40 \mathrm{sec}$ !) in order to converge to an accuracy of $|\Delta f / f|-10^{-4}$ at each grid point; $f$ is any of the variables. The results of their calculations show that the interaction of radiation with soot strongly influences the temperature field and, hence, can influence the circulation.

\section{G. Calculations in three dimensions}

Increases in machine capabilities, together with advances in algorithms, have resulted in the development of three-dimensional codes, as well. Bagnaro et al. (1981) solved the fluid-flow problem in an enclosure with a prescribed fire in three dimensions for the steady-state case, including radiation and combustion. They used a $k-\epsilon$ model for the turbulence. Using a method due to Lockwood and Shah (1976), radiation heat transfer was handled by using a three-dimensional quadratic expression for the radiation intensity $I$, in the approximate radiation transfer equation

$$
(\mathbf{n} \cdot \nabla) I_{\lambda}=K_{\lambda \mathbf{a}}\left[\sigma \mathrm{T}^{4} / \pi-I_{\lambda}\right]
$$

(see Hottel and Sarofim (1967)). The coefficients giving the radiation are functions of position. After integrating Eq.(22) over various solid angles, there result three coupled PDE's in the coefficients (which appear in the function which approximates I). Bagnaro et al. solved this set of PDE's using a three-dimensional version of the program TEACH (Gosman et al.).

Other three-dimensional calculations of fire and smoke spread in rooms have been made in the last few years (see Markatos and Cox (1984), Kumar and Cox (1985), Kumar et al. (1986)). These calculations use the program JASMINE, which is based on a finite-domain solution of the heat-and mass-transfer equations including compressibility. An extensive discussion of the use of UNDSAFE and UNDSAFE II has recently been given by Galea and Markatos (1987). 
A three-dimensional version of UNDSAFE has been developed (Satoh et al. 1980; Satoh et al. 1984), which uses an improved tridiagonal matrix solution scheme (Yang, 1987). Also, as noted earlier, Baum and Rehm (1984a) have performed three-dimensional time-dependent computations using their model.

\section{H. General-purpose programs}

Most of the programs mentioned so far have been developed with a specific application in mind. A number of sophisticated, general-purpose computer programs have been developed which permit the solution of various kinds of equations (algebraic, ODE's, PDE's), or coupled sets of any of the above. Shampine and Watts (1984) discuss software available for solving ODE's; Boisvert and Sweet (1984) describe PDE solvers, as does Walsh (1978). Solvers for linear and nonlinear sets of algebraic equations are discussed by Reid (1978) and by Duff (1984). There are various compendia of available mathematical software, such as IMSL or NAG; these are commercial packages available at most research institutions. There is also software in the public domain, such as LINPAC (Iinear equations solvers), DEPAC (ODE solvers), etc. A text which discusses these and others is that of Kahaner et al. (1989).

There also are commercial codes available now which have been specifically designed to solve fluid-flow problems. Upon developing the $k-\epsilon$ model of turbulence, spalding embedded it in a general-purpose fluid dynamics code which is comercially available as PHOENICS*. Comparable codes such as FLUENT*, NEKTON*, and FIDAP* exist, and there are doubtless others available. A brief description of these, including a very brief description of the discretization and solution methods, as well as turbulence models, is given by Hutchings and Iannuzzelli (1987, 1987a).

These codes have been applied to fire problems in an enclosure (see the work of Cox, Markatos, and coworkers). In principle, they can be applied to multiple rooms; as was stated earlier, however, the limited number of nodes available, and the correspondingly coarse resolution, especially for threedimensional configurations, makes such application, at best, extremely costly.

\section{ZONE MODELS}

\section{A. Description of models}

In a zone model, the space is divided into a small set of large control volumes, with conditions (generally) uniform throughout each. The heat and mass transfer between zones can then be calculated, using the appropriate equations. Generally a different set of processes dominates in each zone. For a single room, the zones which are usually chosen are an upper (hot) region and a (cooler) lower region, and such models are referred to as "twozone" models. This is somewhat misleading, because other zones are also often included: a combustion zone; the walls; the region around vents; and one or more significant objects in the compartment. The thermal plume which develops at and above a fire is another zone; its volume, however, is (incorrectly!) never removed from the lower layer. The plume carries mass, species (inert plus the products of combustion), and enthalpy from the lower layer into the

* Mention of these does not constitute an endorsement, implicit or explicict, by the National Institute of Standards and Technology. 
upper one. When the fire lies within the upper layer, however, no mass is carried up from the lower layer. Note that the upper and lower layer are zones whose volumes vary, in distinction to field model cells.

Field models yield the velocity and temperature distributions (although the resolution and accuracy of the results depend on the grid size); zone models do this only crudely. The geometry of the room and its furnishings can have large effects on the nature of the recirculation patterns; hence the higher spatial resolution of field models can sometimes be important. For example, detailed knowledge of the temperature and/or flow field near some critical item (such as a smoke detector or sprinkler) may be necessary. Also, fluiddynamical considerations are automatically built into field models, rather than being forced into oversimplified approximations. Thus, field models follow the movement of the thermal plume, rather than assuming that deposition of mass and energy from the plume/combustion zone into the upper layer is instantaneous. Similarly, they describe the spread of the ceiling jet to the entire upper layer, rather than assuming instantaneous mixing.

On the other hand, field models have certain drawbacks: they strongly emphasize the fluid-flow aspects of the fire problem, but generally give correspondingly little weight to two other classes of processes central to understanding fire spread and fire behavior: solid-phase processes (heating, pyrolysis, ignition, charring), and combustion processes. There are other good reasons, as well, to believe that zone models are often preferable to field models, in the fire area. They will not be listed, however, in order to avoid the appearance of making this a polemical tract. It should be pointed out, nevertheless, that due to the vast computer requirements, it is not now practical -. nor does it seem likely to become so in the near future -. to describe fires in multiple-room enclosures with a field model; zone models are the only ones available for that purpose at present.

In order for any fire model to be truly useful, it will have to include phenomena which are not so well understood today. Progress needs to be made, for example, in understanding the behavior of wall fires, of burning in vitiated air, of extinguishment, the production of $\mathrm{CO}, \mathrm{HCN}$, and other toxicants, etc.

Even when the basic equations for some process are either not known or not solved, useful correlations may exist that can be used in a zone model. Thus, many global phenomena can be included in zone models which are not easily incorporated in field models. For these reasons, a great deal of effort has gone into the development of zone models. In one sense, zone models may be thought of as hybrids: field models solved via finite-volume methods, where the cells are very large, and where, as a result, a great deal of phenomenological subgrid modeling replaces expensive detailed simulation performed with marginal resolution at best.

Zone models can be classified in several ways. We will begin by considering steady-state vs transient (time-dependent) models:

1. Steady-state models

These models span the spectrum of sophistication and complexity from a relatively simple compendium of individual equations yielding estimates of upper-layer temperatures in a single room, time to flashover, etc. (Lawson et al., 1985; Nelson, 1986), to quite sophisticated models which take many physical phenomena into account and solve the equations of conductive, radiative, 
and convective heat transfer from first principles (Quintiere, 1977). In between are models such as COMPBRN (Siu 1982, Chung et al. 1984), which uses many empirical correlations, as well as basic equations, to describe the results of various physical processes. The earliest model (Kawagoe, 1958) treated the steady state.

Since time derivatives vanish, these models consist of sets of coupled algebraic equations. The solution of sets of algebraic equations was discussed in section III.E; further discussion is given in section IV.D.

\section{Transient models}

There are single- and multiple-room models. For comparisons among some of these models, see Parikh et al. (1983), Mitler (1985a). As is the case for field models, these models have many features in common. They are mostly twozone models, with similar plumes and vent-flow descriptions. Many of them include the radiative exchanges between gases and solids, and some calculate the heating of target objects.

\section{B. Formulation of the problen}

Only a small subset of the existing models will be examined. Primary emphasis will be placed on the Harvard family of models, with which the author has greatest familiarity. These, and the model FAST, use prototypes of the numerical methods used in most zone models.

1. The model FAST

Most of the models solve a relatively small set (about five per room) of coupled, generally nonlinear, $O D E^{\prime} s$ which describe the bidirectional flow of gases through vents, the rate of change of mass and temperature in the upper layex, etc. For example, FAST (which is based on BRIl (Tanaka, 1983)) solves the equations

$$
\begin{aligned}
& \dot{\mathrm{P}}=s /(\beta-1) \mathrm{V}, \\
& \dot{\mathrm{T}}_{i}=\beta^{-1}\left(\mathrm{~T}_{1} / \mathrm{PV} \mathrm{V}_{1}\right)\left[\dot{\mathrm{E}}_{1}+\mathrm{V}_{i} s /(\beta-1) \mathrm{V}\right],
\end{aligned}
$$

where $\quad i=U, L \quad(U=$ upper, $L=$ lower $)$

and $\quad \dot{V}_{U}=\left(c_{p} \dot{m}_{U} T_{U}+\dot{E}_{U}-V_{U} s / V\right) / P \beta$.

Here $s=c_{p} \dot{m}_{U} T_{U}+c_{p} \dot{m}_{L} T_{L}+\dot{E}_{y}+\dot{E}_{L}$

and $\quad \beta=c_{p} / R=\gamma /(\gamma-1)$;

$P, V, T, R, c_{p}$, and $\gamma$ are, respectively, pressure, volume, temperature, the gas constant, specific heat, and $c_{p} / c_{v}$; see jones (1985) for the rest of the nomenclature. The right-hand sides of these equations are the source terms. The rooms are most strongly coupled through the pressure equations, which determine the flows through vents connecting the rooms, and to the exterior.

2. The model Harvard Mark 5

Mark 5 is a single-room model in which a small set of ODE's describes the rate of change of what may be called the "primary" variables. These are the mass and energy of the two layers, the size of the fire if it is growing, and the pressure at the center of the floor. This set is supplemented by a PDE 
which describes heat diffusion through solids, yielding the temperatures of walls and heated objects. It is also supplemented by a number of algebraic equations involving other variables, such as the radiation flux from the hot layer to any object or wall, the convective heating/cooling of walls, the pressure drop across any vent produced by the fire (i.e., the buoyancy-induced flows), etc.

Mark 5 takes into account not only fluid flows, but other dynamics of the fire, e.g., the radiative feedback from the room to a pyrolyzing (burning) surface, the resulting spread of that fire on the surface, etc. Therefore many more variables must be taken into consideration than in FAST or the Consolidated Compartment Fire Model (CCFM; see Cooper and Forney, 1987). The additional variables may be thought of as auxiliary variables, but are clearly of interest for an accurate description of a compartment fire.

The plumes are described by equations which give the mass and enthalpy injected into the upper layer due to a source in the lower layer. Several choices are available in the program; the simplest is the point-source plume of Morton et al. (1956). The mass flow into the upper layer from a thermal plume of height $x$ is given by

$$
\dot{\mathrm{m}}_{\mathrm{p}}=\rho_{0}\left[\left(4 \cdot 3^{5} \pi^{2} \alpha^{4} g \dot{\mathrm{Q}} \mathrm{x}^{5}\right) /\left(5^{4} c_{\mathrm{p}} \rho_{0} \mathrm{~T}_{0}\right)\right]^{1 / 3}
$$

where $\rho_{0}$ and $T_{0}$ are the ambient density and temperature, $g$ the acceleration of gravity, $\dot{Q}$ the convective heat release rate, and $\alpha$ is the entrainment constant (for turbulent entrainment of air into the plume). The resulting energy transport into the upper layer is

$$
\dot{E}_{p}=\dot{m}_{p} c_{p} I_{0}
$$

Vent flows are given by a hydrodynamic approximation to the fluid flow equations, and are described by Eqs.(37)-(39), below. In order to follow the transient heating up of walls or "target" objects, the heat diffusion equation

$$
\frac{\partial T}{\partial t}=\alpha \frac{\partial^{2} T}{\partial x^{2}}
$$

which is a PDE, must be solved (assuming that heat diffuses internally only by conduction). Here it is assumed that

(a) The thermal conductivity $k$, the specific heat $c$, and the density $\rho$ are constant (independent of $x, t$, and $T$ ). Hence so is the thermal diffusivity

$$
\alpha=\mathrm{k} / \rho \mathrm{c} \text {. }
$$

(b) The flow of heat is one-dimensional (slab geometry), and

(c) there are no sources or sinks of heat in the solid.

The numerical solution is given by Eqs. (65)-(70), in section IV.E.1. A flammable material is taken to ignite when its surface reaches an ignition temperature. There are three kinds of fire algorithms built in: a growing fire, a pool (constant size) fire, and a burner fire. The burning rate of a pool fire depends on the flux incident on its surface, and therefore on the temperature and emissivity of the hot layer, of the ceiling and walls, and on the radiation from flames from other fires as well as from its own. The burner fire, on the other hand, is unaffected by external fluxes; it is prescribed by the user. Finally, the growing fire is like the pool fire, but the radius is permitted to increase in size. The equation for $R(t)$ is 


$$
\dot{R}=A C\left(1+C / 2+C^{2} / 3\right)
$$

where $A$ is the "growth rate parameter," $C \equiv \phi / \sigma T_{f}^{4}, \phi$ is the net flux falling on the surface, and $T_{f}$ is the mean flame temperature.

Species production rates are evaluated simplistically, as a constant fraction of the pyrolysis rate. Oxygen consumption is found in the same way; then the rate of change of species $i$ in the upper layer is readily found from the equation

$$
\dot{\mathrm{m}}_{1}=\dot{\mathrm{m}}_{\mathrm{f}} f_{1}-\dot{\mathrm{m}}_{\mathrm{q} v} \mathrm{Y}_{1}
$$

where $\dot{\mathrm{m}}_{f}$ is the fuel flow rate, $\dot{\mathrm{m}}_{\mathrm{uv}}$ is the rate at which hot gases leave the layer through the vent(s), $Y_{1}$ is the concentration of species $i$ in the layer, and $f_{1}$ is the "yield" of species $i$, in $\mathrm{kg} / \mathrm{kg}$. For oxygen $f_{0}$ is negative, and another term is added to account for the injection of oxygen into the upper layer through the plume.

Once the soot, $\mathrm{CO}_{2}$, and $\mathrm{H}_{2} \mathrm{O}$ concentrations are known, the absorption coefficient of the upper layer is calculated. This is done in one of two ways. The first is extremely simple: the soot concentration is multiplied by a constant determined from an experiment. The second way includes the absorption by the molecular bands of $\mathrm{H}_{2} \mathrm{O}$ and $\mathrm{CO}_{2}$; the algorithm is rather complex; see Modak (1978/9). Once $\kappa$ is known, the absorption and emission. of radiation by the layer is readily calculated, via a mean-beam-length approximation or better. The emission from flames is calculated assuming that the flame shape is a cone; the emission coefficient of the flame is input by the user. Heat exchange with the walls is by convection and radiation. For example, the convective heating flux to the wall/ceiling from the hot layer is

$$
\phi_{c o n}=h\left(T_{L}-T_{W}\right)
$$

where the temperature-dependent heat transfer coefficient $h$ is taken to be

$$
h=\min \left[a, b+(a-b)\left(T_{L}-T_{W}\right) / 100\right] \text {, }
$$

with $T_{L}$ the layer temperature, $T_{W}$ the wall temperature, and $a$ and $b$ are the maximum and minimum values that $h$ can take on, respectively.

One poor assumption made in this model is that none of the oxygen in the upper layer can participate in the combustion process. Hence if the interface height falls so low that the plume height is insufficient to entrain enough $\mathrm{O}_{2}$ to provide combustion for the fuel, "oxygen starvation" is said to ensue. The complete set of equations used in Mark 5 (i.e., the model) are given in Mitler and Emmons (1981). A slightly earlier version was given in Emmons (1979).

The equation for the pressure entails enough interesting points that it deserves a detailed discussion: When the physical processes which affect the pressure in the room occur on a time scale which is long compared to the transit time of a sound wave in the enclosure, the compressibility of the gases in the room can be ignored; that is, the gas acts as an incompressible fluid. The appropriate equation for the mean pressure at the floor of the room, $\mathrm{p}$, is then (Quintiere, 1981) 


$$
V \dot{p}+\gamma p \underset{1}{i}\left(\dot{\mathrm{m}}_{1} / \rho_{1}\right)=(\gamma-1)\left[\dot{Q}+\sum_{j}\left(c_{p} \dot{\mathrm{m}}_{j} T_{j}\right)\right]
$$

where $\dot{Q}$ is the net heat release rate in the room, $\dot{m}_{1}$ is the mass flow rate out of the room through vent $i$ of fluid of density $\rho_{1}$, while $c_{p} \dot{m}_{j} T_{j}$ is the inflow enthalpy through vent $j$. This equation is very stiff, and slows down the solution of the equation set considerably.

For fires which take place in enclosures which are not hermetically sealed, the pressure stays (nearly exactly) constant. The pdV work done by the gases while the hot layer is expanding (or shrinking) can be a large fraction of the layer energy change; however, in Mark 5, enthalpy is used rather than internal energy, and it turns out that $V d p$ is a very small fraction of the layer energy change. It is therefore ignored; moreover, it is assumed that the equation is so stiff that $\dot{p}$ is vanishingly small. Hence the pressure is found in a quasisteady-state approximation. Thus, the pressure difference at any vent is obtained by noting that at any moment, the flows induced by that pressure difference must yield mass and energy conservation. This is exactly the same approximation as is made by Tanaka in the BRI2 model.

Thus, assuming the ideal gas law, neglecting the differences in molecular weight among air, fuel, and product species, and (finally) neglecting the change in specific heat with temperature, the total internal energy in the room gases remains constant. Thus the rate of change of gas mass in the roorn is

$$
\dot{m}_{R}=\dot{m}_{o u t}-\dot{m}_{i n}=\dot{E}_{L} / c_{p} T_{a}-\dot{m}_{L}+\dot{m}_{f}
$$

where $m_{L}$ and $E_{L}$ are the mass and internal energy of the upper layer, and $\dot{m}_{f}$ the rate at which fuel gas mass is produced in the room, either by pyrolysis or via a gas burner; this is derived in Mitler (1978).

We will now indicate how the mass flow and pressure are related: the pressure at various heights in the room are referred to that at the floor, $P_{f}$. The mass flow through a vent at a given height is calculated by using the hydrostatic approximations to fluid flow. Thus, it is assumed that momentum is conserved at each horizontal plane. The vent is divided into a number of horizontal strips, the number depending on how many changes in density or flow direction are encountered between the bottom and the top of the vent; there can be as many as six. The pressure difference from the bottom to the top of each strip is a linear function of height. Consider strip $i$; if the pressure difference at the bottom (top) of the strip, at height $h_{1}\left(h_{1+1}\right)$ is $\Delta p_{1}$ $\left(\Delta p_{1+1}\right)$, then the flow in that strip is related to the pressure difference across the vent at that height according to

where

$$
\dot{m}_{1}=G_{1} \frac{\left|\Delta p_{1+1}\right|+\sqrt{\left|\Delta p_{1} \Delta p_{1+1}\right|}+\left|\Delta p_{1}\right|}{\sqrt{ }\left|\Delta p_{1}\right|+\sqrt{ }\left|\Delta p_{1+1}\right|}
$$

$$
G_{1}=\left(\operatorname{sign} \Delta p_{1}\right)(2 / 3) C_{d} B\left(h_{1+1}-h_{1}\right) \sqrt{2 g \rho \rho_{a}}
$$

where $B$ is the width of the vent, $C_{d}$ is the orifice coefficient, usually taken to be 0.68 , and $\rho_{a}$ is the density of the incoming (usually ambient) air. The derivation is given in Mitler and Emmons (1981) and in DiNenno et al.

(1988) (Section 1, Chapter 8,. "Vent Flows," by H.W. Emmons). This is a quasi- 
steady-state expression, but is quite adequate, so long as there are no compressibility effects to consider. Given $\Delta \mathrm{p}_{1}$ (the pressure drop at floor height), all the other $\Delta \mathrm{p}_{i}$ 's are fixed, and therefore the $\dot{m}_{i}$ are found. Finally, the mass flows must satisfy mass and energy conservation. The set of equations (38) cannot be inverted (unless the entire vent opening is one strip) so as to give $\Delta \mathrm{p}_{1}$ in terms of the mass flows; if we write

$$
\dot{\mathrm{m}}_{\mathrm{R}}+\dot{\mathrm{m}}_{1 \mathrm{n}}-\dot{\mathrm{m}}_{\mathrm{out}}=\mathrm{f}\left(\Delta \mathrm{p}_{1}\right)=0 \text {, }
$$

it is clear that $\Delta \mathrm{p}_{1}$ is a zero of the function $f$; it is found by a Newton technique.

\section{Structure of the equations}

The equations of the system for Harvard Mark 5 and Mark 6 (the latter is described in section IV.G) are of the form

$$
\begin{aligned}
& 0=\mathbf{f}(\mathbf{u}, \boldsymbol{\nabla}, \boldsymbol{w}) \\
& d \boldsymbol{v} / d t=g(u, v, w) \\
& w=h(u, v, w) \\
& y=e(u, v, w)
\end{aligned}
$$

where $\mathbf{u}, \mathbf{v}, \mathbf{w}$, and $\mathbf{y}$ are arrays ("vectors") of variables. Each variable is an (implicit) function of $t$, the time since the start of the calculation. There is generally no explicit $t$-dependence in any of the functions $\mathbf{f}, \mathbf{g}, \mathbf{h}$, and $e$, which are vector functions of those variables. If the number of components in array $\mathbf{a}$ is represented by [a], then

$$
[\mathbf{f}]=[\mathbf{u}],[\mathbf{g}]-[\mathbf{v}],[\mathbf{h}]=[\mathbf{w}] \text {, and }[\mathbf{e}]=[\mathbf{y}]
$$

must hold. Moreover,

$$
[\mathbf{f}]+[\mathbf{g}]+[\mathbf{b}]+[\mathbf{e}]=\mathrm{N}
$$

where $\mathrm{N}$ is the total number of variables. Equations (41), (43), and (44) are the algebraic equations, and the corresponding variables (the components of $\mathbf{u}$, $w$, and $y$ ) the algebraic variables. The components of $\nabla$, corresponding to the differential equations (42), can be referred to as the "integrated variables."

Eqs.(41) are root-finding equations, and the components of $u$ are the rootfinding variables. Eqs.(43) [cf Eqs.(51), below] are fixed-point equations, and the components of are the fixed-point variables. Finally, in the original formulation of the problem, there may be a subset of variables which depend only on other variables, and not on each other. They are represented by the vector $y$; thus, none of the variables $y$ are found in the arguments on the right-hand side of Eqs.(41) to (44). Those equations represented by Eqs. (44) are also called "eliminated variable" equations, and the components of $y$ are the eliminated variables. They may also be referred to as "auxiliary," or "dependent," variables.

Evidently, Eqs. (43) and (44) could be converted to the form (41), but the chosen formulation is more economical of computer time because a system of implicit fixed-point equations, such as (43), can (usually) be solved by a successive substitution method, which requires fewer operations than the 
Newton method needed for Eqs.(41). Eliminated variables are trivial: one need only solve (41), (42), and (43) simultaneously, and then substitute the resulting values for $u, v$, and $w$ back into (44) to find $y$. Eqs.(41) are the nonlinear algebraic equations for the pressure.

\section{Numerical methods for solving the model equations}

Analytic solutions for these coupled sets of equations are generally not possible. Hence numerical methods must be used. As is the case for field models, the differential equations are replaced by difference equations which are then solved numerically. In the BRI models, the equations are solved explicitly, using explicit Runge-Kutta (R-K) methods (see, for example, Ralston, 1965; Abramowitz and Stegun, 1964). The solution is obtained with R$\mathrm{K}^{\prime} \mathrm{s}$ of increasing order, until the solution of that order agrees with that of the next-lowest order. If convergence does not occur by the time a sixthorder version is used, the time step is cut to one-tenth its original value, and the process repeated. If the solution is still not obtained to the prescribed accuracy, the calculation stops.

The time rate of change $\ddot{p}$ is assumed to be extremely small, and therefore the pressure equation is taken to be an algebraic equation. The pressure equation is solved by using Crout's method to invert the matrix. That gives new pressures which yield new flows; the whole procedure is iterated until convergence is established. One difficulty with BRIl was that the initial values had to be guessed very shrewdly, else convergence could not occur.

Consider the $i$ th member of the fixed-point equations (43); when $w_{i}$ does not appear on the right-hand-side, the equation is explicit, and the solution for $w_{1}$ at any given iteration is exact. When $w_{i}$ does appear on the right-hand side, the equation for $w_{1}$ is implicit, and the solution will generally be only approximate, assuming an approximate value of $w_{i}$ is available for use on, the right-hand-side. Thus, Eq.(41) (that is, Eq. (38) for $\Delta \mathrm{p}_{1}$ ) is, by definition, implicit in $w_{1}$. We could make it explicit if we could invert Eq. (38) and solve it for $\Delta \mathrm{p}_{1}$; as pointed out above, however, this cannot be done in general.

Finding $\Delta \mathrm{p}_{1}$ as the root of Eq. (40) presents two interesting problems: first, any numerical "package" to be used in solving the equations must include a root-finding part. Second, the following interesting conundrum arises: the algebraic equations are solved by a successive-substitution (G-S) method; hence it would appear to be a waste of effort to solve exactly for $\Delta p$ in any of the global iteration cycles, since (a) the solution will differ in each subsequent iteration, and (b) the overall convergence of the procedure should guarantae increasing accuracy. On the other hand, it might be that finding the "exact" solution in each of the global cycles would help to accelerate the overall convergence process. In producing Harvard $V$, the former philosophy was adopted, and $\Delta p$ was found with only one local iteration, rather than iterated to local convergence. The second approach has been used in the developmental version of FIRST; the result is as expected: not only has convergence indeed been accelerated by finding $\Delta p$ "exactly" at each time iteration, but the program now runs to completion in many cases where it would "crash" with the previous approach!

A problem with explicit methods is that the time-step size required is prohibitively short when stiff equation sets are being solved. More frequent- 
ly, therefore, predictor-corrector methods are used to solve such sets of equations. FAST was based on BRII, but the numerical method was much improved. Since the rates of the various dynamical processes vary over orders of magnitude, these equations are stiff, and the time-steps required even by predictor-corrector methods may become prohibitively small. However, FAST uses the Selected Asymptotic Integration Method (SAIM) for this purpose (see Young and Boris, 1977; Oran et al. 1979; Young, 1980), which is a modified predictor-corrector method. In its simplest form, the method may be described as follows: given a set of ODE's of the form

$$
\dot{x}_{1}=F_{1}=Q_{1}-L_{1} x_{1}
$$

where $Q_{1}$ is the formation rate of the quantity $x_{1}$ and $L_{1} x_{1}$ is the loss rate, the equations are separated into two classes: "normal" and "stiff," according to a simple criterion.* For the normal equations at time $t+\delta t$, the first iteration for $x_{1}$, the predictor, is

$$
x_{1,1}=x_{1,0}+\delta t F_{1,0}
$$

where $x_{1,0}$ is the initial value (at time $t$ ); for the corrector steps, the $k+1$ iteration is given by

$$
x_{1, k+1}=x_{1,0}+\delta t\left[F_{1,0}+F_{i, k}\right] / 2
$$

For the stiff equations, the predictor and corrector equations are, respectively,

and

$$
x_{1,1}=x_{1,0}+\frac{\delta t F_{i, 0}}{1+\delta t L_{i, 0}}
$$

$$
x_{1, k+1}=x_{1,0}+\frac{2 \delta t\left[Q_{1, k}-L_{1,0} x_{1,0}+F_{1,0}\right]}{4+\delta t\left[L_{1,0}+L_{1, k}\right]} .
$$

The method works well, and it would be most useful to go through a rigorous stability/convergence/error analysis of the method.

In FAST, the source terms are calculated explicitly at each time step. Thus the initial iterates are based on the values the primary variables have at the beginning of that step, making FAST a single-step model.

The more standard method for solving sets of stiff coupled ODE's numerically is an implicit method using a backward difference formula, such as the Gear method (Gear, 1971). This is a multivariate Newton-Raphson technique (see section IV.E.1). CCFM (Cooper and Forney, 1987) uses a Gear-like package called DEBDF (Differential Equations via Backward Difference Formula) to solve the ODE's. DEBDF is part of a three-routine set called DEPAC developed at Sandia Labs (Watts and Shampine, 1979). These packages are quite sophisticated; the programs internally determine the required time-step sizes as they proceed. Some packages of ODE solvers are so sophisticated as to include a heuristic which decides whether the equations are stiff, or not, and choose internally which solution algorithms to use! (see Radhakrishnan, 1990).

* Canonically, one refers to the entire set of coupled equations as stiff. 
In general, numerical "packages" are used to solve the ODE's; the PDE must be solved separately. A solution method for Eq. (30) is described in section IV.E.1. However, it is possible to avoid having to solve a PDE, by writing the surface temperature as a convolution integral. It can also be done by transforming the PDE into a set of coupled ODE's. This can be done in three ways: first, by using an integral technique to find the surface temperature approximately. Second, by using similarity solutions to extract the spatial dependence analytically, and third, by using a PDE solver such as the method of lines (Aiken, 1985); there is one ODE per mesh point in the solid into which heat is diffusing. This subset of ODE's often produce great stiffness in the overall set, however. Since a relatively fine mesh is used to solve the PDE for all methods but the first, these constitute a field-model approach. Thus the entire model should more properly be called a hybrid (partly zone, partly field) model.

\section{E. Harvard Mark 5}

\section{Solution methods used}

In Mark 5, every variable is calculated so that its value is consistent with that of every other, according to all the available relationships, within the prescribed accuracy. That is, if there are $\mathrm{N}$ variables being followed, there are $N$ algebraic equations to be solved simultaneously. There are 4 $O D E^{\prime} s$ describing the rates of change of mass and energy in the layers, 3 ODE's for each vent, and 5 for each burning object (describing the mass and radius of the fuel packet, the energy release, and the mass and enthalpy injected into the upper layer by the burning object's thermal plume). Hence, for a room with just one burning object and one vent, there are 12 ODE's for 12 primary variables, resulting in 12 (out of the $N$ ) difference equations.

In FAST (Jones, 1985), a set of algebraic relationships similar to, but smaller than, those in Mark 5 are assumed to be the source terms on the righthand side of the differential equations

$$
\dot{x}_{1}=\theta_{i}(x)
$$

where $x_{1}=x_{1}(t)$ is the value of the $i^{\text {th }}$ primary variable, and the vector symbol $\mathbf{x}$ stands for the set $\left(x_{1}\right)$. Equations (52) are essentially Eqs.(42).

Since ODE's are transformed into algebraic equations, there is no difference in principle between the solution method used in FAST and other ODEbased models and that used in Mark 5. In each case, a set of nonlinear algebraic equations is solved numerically.

The numerical calculation procedures are described in considerable detail in Mitler and Emmons (1981). A brief synopsis is presented here: rewrite the (linear or nonlinear) "fixed-point" algebraic equations (43) in the form

$$
x_{k}=g_{k}(x)
$$

The principal method of solution used is the successive-substitution method, in which the variables $\mathbf{x}$ are calculated iteratively. After $k$ iterations, the $(k+1)^{\text {st }}$ value of variable $x_{1}$ is found by setting 


$$
x_{1}^{(x+1)}-g_{1}\left(x^{(k)}\right)
$$

where $\mathbf{x}^{(k)}-\left\{\mathbf{x}_{1}^{(k)}\right\}$ is the set of values found at the previous iteration. The time dependence of each variable is not displayed only in order to make the equations more succinct.

There is one exception to the formulation of the equations all being of the forms (41)-(44): the $\operatorname{PDE}(30)$ is not of any of the forms (41)-(44), and must be solved independently, as discussed earlier.

The calculation for $\Delta p$ is done inside a physics subroutine, since it cannot be carried out in the numerics package. This is so, because the technique (54) is only valid for fixed-point equations, which Eq. (41) [or (40)] is not. Thus, given all the other $x_{1}^{\prime} s$ at the nth (global) iteration, we find a value for $\Delta p$..that is, for $x_{j}^{(n)}$.. in the same way as Eqs. (53) yield values for all the other $x_{1}$ 's. We will therefore include Eq. (41) with the rest of Eqs. (53). Note that in order to be able to find $\Delta p$, we must have already evaluated some of the flow rates, so that this variable is best calculated late in the cycle. This different treatment of $\Delta p$ is an exception to the modularity principle: a program should be structured so that any physics algorithm can be replaced with an improved or corrected version, or a new numerical procedure readily introduced, without requiring extensive program changes. Generally, modularity is best achieved by decoupling the various calculations into independent subroutines, and separating the numerical procedures from the physics.

Next, consider Eqs.(42); they are solved by numerical integration:

$$
x_{1}(t+\Delta t)=x_{1}(t)+\int_{t}^{t+\Delta t} \theta_{1}(x) d t
$$

where $\Delta t$ is the time-step interval. In Harvard Mark 5, the integration method which is used is the trapezoidal rule:

$$
x_{1}(t+\Delta t)=x_{1}(t)+\Delta t\left[\theta_{1}(t)+\theta_{1}(t+\Delta t)\right] / 2
$$

where we have written $\theta(t)$ as a symbolic way to represent $\theta[x(t)]$. Although this is an explicit integration, the overall method, based on successive substitutions, is implicit. Equations (56) are now also of the form (53), and can be included in that set, as well. Thus equations (41), (42), and (53) [i.e., (43)] have all been cast into the form (53), and the successive substitution method can now be applied to them all. Use of the trapezoidal rule seems crude, especially when compared with the methods used in FAST, CCFM, and other models. However, the inherent error is - $(\Delta t)^{3} \dot{\theta}_{i}(\tau) / 12$ per time step, where $t<\tau<t+\Delta t$. Hence the magnitude of the total error in the ith variable (assuming uniform time steps only) is $\left[(\Delta t)^{3} / 12\right] \Sigma \theta_{1}\left(\tau_{j}\right)$, where $j$ goes from 1 to $n=t / \Delta t$. If we approximate the sum by an integral, it can be shown that the cumulative numerical error in $x_{1}$ is

$$
\text { Error } \approx-(\Delta t)^{2}\left\{\dot{\theta}_{1}(t)-\dot{\theta}_{1}(0)\right\} / 12-(\Delta t)^{3}\left\{3 \ddot{\theta}_{1}(t)-\ddot{\theta}_{1}(0)\right\}+0\left([\Delta t]^{4}\right) .
$$

At large times, $\theta_{1}$ and $\dot{\theta}_{1}$ should both go to zero. Thus, knowing the function $\theta_{1}(t)$ [and therefore $\dot{\theta}_{1}(0)$ ], we can readily choose $\Delta t$ small enough to keep the total error within satisfactory bounds. Indeed, note the comment at the end of the Accuracy subsection, below. 
In early versions of the program, the Jacobi method was used to solve Eqs. (53). In Mark 5, a variant of the G-S method is used, instead. An important feature of the Jacobi method is that the results are essentially independent of the order in which the equations are solved. This is not the case for Gauss-Seidel, and a consequence is that some orderings will require more iterations than others, for convergence. Indeed, for some cases one ordering may diverge, while another ordering produces convergence. Define

$$
g_{j, k}=g_{j}\left(x_{1}^{k+1}, x_{2}^{k+1}, \ldots, x_{j}^{k+1}, x_{j}^{k}, x_{j+1}^{k}, \ldots, x_{N}^{k}\right) \text {. }
$$

When Mark 5 was first being constructed,

$$
x_{j}^{k+1}=g_{j, k}
$$

was used. Still faster convergence was obtained with what might be called successive under-relaxation (Smith (1965), chap.2): instead of Eq.(59), $x_{i}^{k+1}$ is now given by

$$
x_{j}^{k+1}=\lambda g_{j, k}+(1-\lambda) x_{j}^{k} \quad \lambda<1
$$

where $\lambda$ is the relaxation factor. Best results were found with $\lambda=1 / 2$. The use of Eq. (60) rather than (59) has sometimes led to the convergence of an otherwise divergent calculation (Mitler and Emmons, 1981). When the method converges, it does so linearly. That is, for all sufficiently large k (i.e., $k$ greater than some value $k_{c}$ ),

$$
\left\|x^{k+1}-x\right\| \leq c|| x^{k}-x \|^{m} \quad k>k_{c}
$$

with $m=1$ and $|C|<1$, where $\mathbf{x}$ is the exact solution at time $t$ and the double bars denote the notn of the vector (Atkinson, 1978). The Gauss-Seidel method has yielded superior results for the enclosure problem than the Jacobi method has: it converges over a wider domain, and usually with substantially fewer iterations.

The starting values at a new time step are given, for each variable, by a quadratic extrapolation of the values of the variable at the last three time steps; this is analogous to the predictor step in predictor-corrector methods. If, during the calculation, a variable is found to fall outside physically reasonable (or permissible) bounds, its value is reset to the value it had at the last time step. This procedure, carried out in sections called LIMITS, is ad hoc and has drawbacks, but it made the calculation still more robust.

The second method used to solve the equations is the multivariate NewtonRaphson technique, normally applied to functions of the form (41). For fixedpoint equations of the form (54), one defines the vector function $f$ :

$$
f(x)=x-g(x) \text {. }
$$

The Newton technique for the case of $N$ simultaneous equations takes the form

$$
x^{k+1}=x^{k}-J^{-1} f\left(x^{k}\right)
$$

where $J$ is the $N x N$ Jacobian matrix $\left\{\partial f_{1} / \partial x_{j}\right\}$. For a single variable, a sufficient condition for convergence is that 


$$
\left|f\left(x^{k}\right) f^{\prime \prime}\left(x^{k}\right) /\left[f^{\prime}\left(x^{k}\right)\right]^{2}\right|<1
$$

for all k (Scarborough (1962), para. 78). In Mark 5, the equations are solved by Gaussian elimination with partial pivoting (Scarborough (1962), p.270; Smith, 1965) using the code of Forsythe and Moler (1967). If an exact Jacobian were known at each iteration, then the algorithm given by Eq. (63) would converge quadratically, i.e., with $m=2$ in Eq.(61). The finite difference approximation to the Jacobian used in Mark 5 is not exact, so that in fact we are using a secant method. It can be shown that this leads to a rate of convergence given by $m=1.839$, in a single variable (Jarrat, 1970). When the condition number is very large, the Newton method will not work properly; what can be done about this is discussed below, in subsection IV.G.

The full Newton method is quite time-consuming, and some alternatives were devised. If $J(t)$ varies slowly enough, it requires recomputation only once every few time steps. This simple approach is called Newton Super Fast, and is referred to as NWSF. It works much of the time, and is indeed very much faster than the multivariate Newton method (NWTN).

Using a numerics "package" which solves sets of simultaneous algebraic equations (or ODE's and algebraic equations) will not suffice for the PDE (30) which describes heat diffusion. Hence we must again deviate from modularity, and use a numerical procedure inside a physics subroutine. The technique used in Mark 5 to solve for the surface temperature as a function of time is a simple explicit finite-difference method: the wall (or object) is divided into $M$ slabs of thickness $\delta \mathrm{x}$ by planes parallel to the surface. The temperature profile within the wall is found at successive time steps. The Courant criterion is satisfied when

$$
\delta \mathrm{x} \geq(\delta \mathrm{x})_{\min }=\sqrt{2 \alpha(\Delta t)_{\max }}
$$

where $(\Delta t)_{\max }$ is the largest time step taken in the problem and $\alpha$ is the thermal diffusivity (assumed to be constant). Thus $M$ is given by

$$
M=\left[\theta /(\delta \mathrm{x})_{\min }\right]
$$

where $\theta$ is the wall thickness ([a] means "the integer part of $a$ "). Then the temperature at the front surface, at time $t+\Delta t$, is given by

$$
T_{1}(t+\Delta t)=T_{1}(t)(1-a \Delta t)+a \Delta t\left\{T_{2}(t)+b \phi_{1}(t)\right\}
$$

Here $\phi_{1}$ is the net heating flux to the front surface, $\Delta t$ is the current value of the time step, $T_{1}$ and $T_{2}$ are the temperatures at the first and second nodes (front surface of wall and front surface of second slab, respectively), and a and $b$ are defined by

$$
a=2 \alpha /(\delta x)^{2} \quad \text { and } \quad b=\delta x / k \text {, }
$$

where $k$ is the thermal conductivity. For nodes 2 to $M$, the temperature is given by

$$
T_{1}(t+\Delta t)=T_{1}(t)(1-a \Delta t\}+a \Delta t\left\{T_{1-1}(t)+T_{1+1}(t)\right\} / 2 .
$$

The temperature at the back surface is 


$$
T_{M+1}(t+\Delta t)=T_{M+1}(t)(1-a \Delta t)+a \Delta t\left\{T_{M}(t)+b \phi_{M+1}(t)\right\}
$$

A derivation of these equations is given in Mitler (1978) (also see Chapter 3 in DiNenno et al., 1988).

The overall computation proceeds as follows: the calculation runs along using G-S until it fails to converge at $t+\Delta t_{0}$ in a fixed, user-determined number of iterations, with 35 as the default value. The program thereupon cuts the time step in half and starts over (i.e., tries to converge to $\left.t+1 \Omega t_{0}\right)$, using newly extrapolated values from the previous time steps; this guarantees a starting solution closer to the correct answer, which helps to lead to convergence. If it then succeeds in converging, the calculation continues with $\Delta t=\Delta t_{0} / 2$ for ten steps (with some exceptions), then doubles the time increment, since the difficulty may have only been temporary. Failure to converge in 35 of these smaller steps, on the other hand, causes another halving of the time step, to $\Delta t_{0} / 4$; and so forth. The time step will repeatedly be halved until the solution converges at some point, or until $\Delta t=$ $\Delta t_{0} / 16$, where $\Delta t$ is the newly cut time step and $\Delta t_{0}$ the originally chosen time step, whichever comes first. If the procedure fails to converge at this (smallest) time step, the program switches to NWTN. The program tries to take one step in NWTN, using the time step $\Delta t$, and then (if it converges to a solution) switches to NWSF. The solution mode will remain NWSF until it ceases to converge (in which case it goes back to NWTN, and we start over), or until the size of the time step just taken is $\geq \Delta t_{0} / 4$, in which case it switches back to the G-S mode. If it takes a number of steps successfully in G-S mode, $\Delta t$ is doubled when the total number of time steps taken (from the beginning) is a multiple of 10 . This doubling procedure continues until $\Delta t=$ $\Delta t$ 。 again, where it stays, or until trouble occurs again.

\section{Convergence criterion}

The convergence criterion used is that two successive iterations do not change the value of any variable by more than some small, specified fraction. Since we want the relative accuracy to be about the same for all variables, we normally would demand that

$$
\left|\Delta \mathrm{x}_{1} / \mathrm{x}_{1}\right|<\epsilon_{\mathrm{c}}
$$

for all $i$, where $\Delta x_{i} \equiv x_{1}^{k+1}-x_{1}^{k}$. However, when a variable $x_{1}$ takes on a value $x_{1}$, min which is so small that it ceases to have physical significance, the program stops testing it for convergence. When it takes on a value greater than but comparable to $x_{1, m i n}$, we do not need the full accuracy given by Eq. (71), and it is replaced by

$$
\left|\Delta x_{1} / x_{1}\right|<\max \left\{x_{1, \min } /\left|x_{1}\right|, \epsilon_{c}\right\} \text {. }
$$

The largest value of

$$
\left|\Delta x_{i} / x_{i}^{k+1}\right| \cdot\left[\epsilon_{c} / \max \left(x_{i, m i n} /\left|x_{i}\right|, \epsilon_{c}\right)\right]
$$

is then called the NORM at this iteration. When NORM $<\epsilon_{c}$ for some iteration, the program has "converged" to a solution. 
Suppose the $j$ th variable is the small difference of two large numbers. Then in order for $\mathrm{x}_{\mathrm{j}}$ to be computed to within $\epsilon_{\mathrm{c}}$, its large constituents must be computed rather more accurately. However, this also implies that if we take $\epsilon_{c}$ too small, truncation and round-off errors become significant and it may be literally impossible to obtain "convergence." Hence $\epsilon_{c}$ must be judiciously chosen. The value $\epsilon_{c}$ is user-chosen, with a default value of $3 \times 10^{-4}$. This value is small enough to give satisfactorily accurate answers, but large enough to permit rapid convergence in single-precision arithmetic while avoiding the truncation errors just described.

\section{Scaling of variables}

The physical variables in Mark 5 range in magnitude from energies on the order of $10^{7}$ Joules, to masses on the order of $10^{-5} \mathrm{~kg}$. For a variety of reasons, it is difficuit to work numerically with sets of variables that range, as these do, through twelve or more orders of magnitude. One problem comes about as a result of the finite accuracy of the computer's floatingpoint representation of numbers; for example, in estimating a partial derivative according to the finite difference $f(x+h)$ - $f(x)$, the perturbation $h$ cannot be too much smaller than $x$ in magnitude when $x$ is already small, or the machine will compute the resulting difference as 0 or at best inaccurately. Again, in order to have maximum accuracy in solving the simultaneous (linear) equations in the Newton mode, we need to have all the variables of comparable size. This is accomplished by normalizing all variables by their then-current values, to unity, whenever the transition is made to the NWTN mode. Then the derivatives for the Jacobian are estimated numerically.

\section{Accuracy}

The smaller we make $\epsilon_{\mathrm{c}}$, the more accurately we should converge to an internaliy consistent solution of the algebraic equations. Likewise, the smaller we make $\Delta t_{0}$, the basic time increment, the smaller will be the errors arising from the use of numerical approximations of various sorts. There are some limitations, however. First, the smaller we make $\epsilon_{c}$, the more iterations usually have to be taken in order to converge. Moreover, the more likely we are to fail to converge at all. Indeed, as pointed out above, if we demanded too much precision we would run into round-off and truncation errors, and probably could never converge. The same is true of $\Delta t_{0}$.

Consider the overall (rms, fractional) error per step. The mean convergence error will be $\beta \epsilon_{c}$, with $\beta$ of order unity, and the errors will be assumed to be random. If the numerical error $\left(\epsilon_{n}\right)$ is also random and therefore incoherent with the convergence error $\beta \epsilon_{\mathrm{c}}$, the typical error in one step will be

$$
E_{1}=\sqrt{\epsilon_{\mathrm{n}}^{2}+\beta^{2} \epsilon_{\mathrm{c}}^{2}} \text {. }
$$

The total error after $k$ steps, again assuming random errors, should be about

$$
E_{\mathrm{k}} \simeq \sqrt{\mathrm{k}} \mathrm{E}_{1} .
$$

For a given time step $\Delta t_{0}$, the (fractional, rms) numerical error is $\epsilon_{\mathrm{n}}$, which is proportional to some power of $\Delta t_{0}$ :

$$
\epsilon_{\mathrm{n}}=\alpha\left(\Delta t_{0}\right)^{3}
$$


If the basic time increment is $\Delta t_{0}$, then for a run of duration $t$, the number of time steps will be

$$
k \geq t / \Delta t_{0} \text {, }
$$

and therefore

$$
E_{\mathrm{k}} \geq \sqrt{t} \sqrt{\alpha^{2}\left(\Delta t_{0}\right)^{2 s-1}+\beta^{2} \epsilon_{c}^{2} / \Delta t_{0}}
$$

The value $\epsilon_{c} \approx 3 \times 10^{-4}$ was chosen after numerical experiments on a VAX showed that the final answers (for a 500-sec run of a standard problem) did not differ by more than a few percent for this value, compared to calculations with smaller $\epsilon_{c}^{\prime}$ 's. A series of runs with $\Delta t_{0}=2,1,1 / 2,1 / 4$, and $1 / 8 \mathrm{sec}$ was made, with $\epsilon_{c}$ kept at $3 \times 10^{-4}$. It was found that all the variables appear to converge to asymptotic values as $\Delta t_{0} \rightarrow 0$. Indeed, the deviations from those values were very nearly linear with $\Delta t_{0}$ :

$$
x_{1}\left(\Delta t_{0}\right) \approx x_{1}(0)\left[1+\alpha_{1} \Delta t_{0}\right] \quad \text { all i }
$$

Hence the fractional rms error at time $t$ must be

$$
E(t) \approx\langle\alpha(t)\rangle \Delta t_{0} \text {, }
$$

where $\langle a\rangle$ is the rms value of the $\alpha_{1}$ 's. Only for $\Delta t_{0} \geq 2 \mathrm{sec}$. does the relationship (78) begin to fail. Since the values still appear to be converging toward the exact answer with $\Delta t_{0}=1 / 8$, it appears that:

(a) the value of $\Delta t_{0}$ for which we get minimum error is less than $1 / 8 \mathrm{sec}$, and

(b) $\beta \epsilon_{\mathrm{c}}$, which is independent of $\Delta t_{0}$, must be much smaller than $\epsilon_{\mathrm{n}}$ even for $\Delta t_{0}=1 / 8$.

Therefore we shall take $E_{1} \simeq \epsilon_{n}$ henceforth. If the numerical errors are random, then

(c) the linearity with $\Delta t_{0}$ in Eq. (79), plus Eq. (77) suggests that $s=3 / 2$.

A similar analysis was carried out assuming that the numerical errors are systematic, rather than random. In that case, Eq.(79) implies that $s=2$. Finally,

(d) Eq. (78) appears to show that use of the trapezoidal rule for integration is not the principal source of numerical errors, since that error varies as $\left(\Delta t_{0}\right)^{3}$ for one time step, and as $\left(\Delta t_{0}\right)^{2}$ overall, according to Eq. $(57)$, rather than linearly, as in Eq. (78).

\section{Numerical problems}

The principal numerical problems with Mark 5 were of two kinds: first, the program would not run to completion, some 10.15\% of the time. Second, when the program fails to converge in the G-S mode, it switches over to the Newton method; however, that overcame the difficulty only some $50 \%$ of the time.

Analysis has shown that when a numerical difficulty arises, it most likely is due to some discontinuity inadvertently built into the program (that is, the physics was badly modeled). When such a discontinuity was removed, the numerical problem would disappear. 
Moreover, discontinuities in first derivatives can be troublesome with the Gauss-Seidel method, and deadly with the Newton method since, when the left derivative is different from the right derivative, the Jacobian is not welldefined at the transition point. Finally, as suggested by Eq.(64), it is desirable to have the functions at least twice differentiable. Again, the cure is to avoid modeling a physical process with an expression which has discontinuities in $f^{\prime}$. If such are found, one ad hoc way to remove them is to round the function in that region.

For many problems, the solutions will approach asymptotic values after a certain time. One would suppose that the solver would then zip through the problem in record time. In fact, one sometimes finds that a program will fail in this regime. The reason is that when the variables approach convergence at the prescribed accuracy, it is often because of stiff competing processes. The small differences between relatively large numbers are basically "noise," and hence the Jacobians which are calculated are very poor. The result is an amplification of the noise. Some of the oscillations will be damped out by the solver, but others continue to grow and drive the solution outside of the region of convergence. A simple solution to this problem lies in specifying that differences which fall below the noise level (essentially, below the prescribed error tolerance) be set to exactly zero.

\section{F. The model FIRST}

\section{Improvements over Mark 5}

A number of improvements, mainly in the physics, have been made to Mark 5; the resulting program is called FIRST. These include the effects of layer mixing at vents due to the formation of a turbulent shear layer at the interface, the possibility of having forced ventilation, and a number of other items. The complete list of improvements is too long to give here; it is given in Appendix $C$ of Mitler and Rockett (1987).

FIRST also incorporates several improvements in the numerics: the program is now run in double precision, which minimizes problems arising from working with small differences of large numbers. Indeed, this one change alone to Mark 5 lowered the "bomb-out" rate to something like $20 \%$ of the previous rate; i.e. to $2-38$. It was also realized that one source of difficulties was the "machine $\epsilon$ " problem, and this was removed. The integrations shown in Eq. (55) are now carried out in a separate subroutine, so that the integration technique can easily be upgraded to Adams-Moulton, Simpson's, Weddle's, etc.

\section{Further contemplated changes}

The use of LIMITS (see remarks above Eq.(62)) to help obtain convergence is an undesirable procedure: it can lead to discontinuities, spurious results, and mask errors and inconsistencies in the program. FIRST has had these removed from some of the subroutines. Newer versions of FIRST currently under development have had the numerics improved in a number of ways, including the removal of the LIMITS section from most of the remaining physics subroutines.

Consider the values of the $i^{\text {th }}$ variable at the current $(t+\delta t)$, previous $(t)$, and previous-but-one $(t-\Delta t)$ times. Then in the expression 


$$
x_{1}(t+\delta t)=x_{1}(t)+\omega\left[x_{1}(t)-x_{1}(t-\Delta t)\right] \delta t / \Delta t
$$

which can be used to make an initial estimate of the value of $x_{1}$ at a new time step, $\omega=0$ corresponds to no extrapolation and $\omega=1$ to linear extrapolation. A scheme which merits investigation is the following: numerical experiments can show what value of $\omega$ gives the best convergence for a series of runs. That value can then be used as the default value, but the user would have the option to change the value of $\omega$ if he/she so wished.

Yet another technique which should be tried, is to accelerate convergence of the iterations by some method such as Aitken's $\delta^{2}$ procedure (Henrici (1964), p.70); that method is valid if the sequence of iterates is converging with an error which is approximately in geometric progression.

\section{G. An alternative method}

As was pointed out earlier, the Newton-Raphson method will fail when the Jacobian matrix is ill-conditioned. Nash (1979) discusses the unconstrained minimization problem (Chapters 15-17) and shows that variable metric (quasiNewton or matrix iteration) algorithms are better than the Newton method. He shows that a variant of the Levenberg-Marquardt method (Levenberg, 1944; Marquardt, 1963) is very fast and extremely robust. When det $J$ is "large," the method approaches the Newton algorithm, with rapid convergence. When det $J$ is small, however, then rather than failing altogether, the method approaches the steepest descents algorithm, which still converges, although only linearly. This method has not yet been implemented in FIRST, however.

\section{H. Harvard Mark 6}

Harvard Mark 6 (referred to as Harvard 6, as Mark 6, or as CFC VI) was created as a multi-room version of Mark 5 (Gahm 1983, Rockett and Morita 1985, Rockett et al. 1989). The physical content is essentially the same. One design goal for Mark 6 was to construct the system of equations so as to eliminate as many variables as possible (see section IV.C). Emmons (1978) showed that the order in which the equations are solved greatly influences the apparent number of dependent variables. Ramsdell (1981) devised an algorithm which finds the ordering yielding the minimal set of independent variables and wrote JUGGLE, a computer program which implements this algorithm (198la). However, the program was written in PASCAL, and is too large to be used as a subroutine in Mark 6. It has been used to find the best ordering for the default set of variables.

Equations (41)-(44) are solved using the Gear (1971) ODE/algebraic equation solver. The solver had to be generalized somewhat, to handle Eqs.(41). The ODE part uses an implicit method; the program decides what the time-step size should be as it goes along, based on estimates of the local truncation error, and what order of integration/extrapolation to use. It handles stiff equations relatively well. It is a predictor-corrector method; the algorithm reduces the integration problem to that of solving a system of nonlinear algebraic equations. Solving this system of equations is called the "correction," and is done via a multivariate Newton's method which requires an estimate of the Jacobian matrix. This is not done at every time step, but only when the corrector fails to converge or when 30 corrections have elapsed 
since the last estimation of the Jacobian; this saves computing time. Even when the Jacobian is estimated, it is not the full Jacobian which is estimated: first, the structure of the equations implies that the Jacobian columns for the eliminated variables are known a priori: they are zero except for one's on the main diagonal. Therefore the U/L decomposition only needs to be applied to the upper left-hand corner matrix, whose dimension is given by the number of non-eliminated variables in the system, $[\mathbf{u}]+[\mathbf{v}]+[\boldsymbol{w}]$. Thus, the algorithm first solves for the non-eliminated variables using that portion of the Jacobian. It then uses back-substitution with the remainder of the Jacobian to find values for the eliminated variables.

Second, the algorithm often does not even evaluate the Jacobian for all of the non-eliminated variables. When the off-diagonal entries of $\mathrm{J}$ are small, one can often obtain convergence in the corrector by assuming that those smalI entries are exactly zexo. In this case, $J$ is estimated by assuming it to be the identity matrix except in those colums corresponding to root-finding variables: For those variables, it is seldom true that the off-diagonal entries are small compared to the diagonal ones. Therefore, these columns of $J$ are estimated in the usual manner. In this case, only the upper left-hand corner [f]x[f] matrix need be subject to U/L decomposition. All of the remaining variables can be found by back-substitution. Consequently, this technique is by far the least expensive correction technique. Note that when this simplified Jacobian is used, the resulting correction method has a successive-substitution form. The package does not handle PDE's, and so, just as in Mark 5, the calculation of the heating of objects and walls is handled independently of the general numerical package.

The calculation switches back and forth between the Newton-like technique and the successive-substitution-like technique. It uses the latter (faster) method until the corrector fails to converge at twice the minimum time step and then switches over to the more robust method until the faster method begins to work again. On the other hand, since the faster method is less stable, the integrator tends to take more time steps when it is in use, cancelling much of the savings from the smaller Jacobian decomposition. Moreover, it is not clear that the successive-substitution method will work for systems of equations which include other root-finding equations, as the upper left-hand sub-matrix may becone singular.

The integrator cannot guarantee a solution to the system of equations unless the system satisfies certain conditions (see Gahm, 1983). In part because these are not always satisfied, the program fails to converge, about $10 \%$ of the time. However, one can usually get around such a difficulty by making a small change in the input, which has no real physical significance (such as making a room which is 3.6 meters long, into one which is $3.61 \mathrm{~m}$ long, for example); just one such change will work, 908 of the time, and the result is that the program will work, 998 of the time. Occasionally a calculation will reach a "sensitive" point, where the time steps taken will be the smallest permitted (100 microseconds, as the default) for some time, making the total computing time quite long. The same, of course, is true of most such computer programs.

\section{Validation}

The results of using Mark 5 are discussed in a number of places (Emmons 1979, Mitler and Rockett 1981, Rockett 1982, 1984; Rockett et al. 1983, 
Blomqvist and Andersson 1984, Mitler 1985). The model has been found to predict the outcome of a number of fires reasonably well. Also see Parikh et al. (1983) and Mitler (1985a). A number of studies of the use of FIRST have also been carried out (McCaffrey and Dagher 1989, Duong 1990, Gross and Davis 1988, Todd and Ryan 1989). As might be expected, FIRST is generally an improvement over Mark 5. The results of using Mark 6 are discussed by Rockett and Morita (1985), Rockett et al. (1989).

\section{CONCLUDING REMARKS}

Zone models, at present, allow for a wider range of processes to be incorporated than field models do, while requiring much less computer time or memory -. minutes ys. hours. On the other hand, the capacities of computers are increasing at such a pace that three-dimensional field models with radiation and combustion are becoming possible. Moreover, new numerical techniques and refinements are still appearing for the solution of PDE's.

The numerical techniques currently used in zone models differ among each other, although some variant of a multivariable Newton-Raphson technique is the most commonly used one. It is not yet clear whether it is more expedient to solve a set of differential and algebraic equations, or a set of differential equations with very complicated sources. The nonlinear Gauss-Seidel technique, with certain improvements, works very well, and other possibilities (such as accelerating convergence, using the Levenberg-Marquardt method, etc.) exist. A powerful technique for solving sets of $O D E^{\prime}$ 's which is not very well known, based on a Taylor series expansion of the functions, is a program called ATOMFT (Corliss and Chang, 1982); perhaps its use in this context should be examined.

Fire modeling is in the midst of a vigorous growth phase. Efforts are being made in at least a dozen places throughout the world to develop perhaps double that number of models. Although progress is being made, these are isolated efforts, with a good deal of repetition and overlap. New programs do not profit from others nearly as much as is desirable, so "the wheel is (continually) being reinvented." Further, each individual effort is hampered by local limitations of time, manpower, and money. Progress - - especially in incorporating more physical and chemical phenomena in the models -. would be much faster if the same resources now being applied to more than twenty efforts were applied to a much smaller number -- perhaps four or five. That would still allow for alternative avenues to be explored.

\section{REFERENCES}

Abramowitz, M., and Stegun, I.A., eds. (1964), Handbook of Mathematical Functions, Applied Mathematics Series, No.55; National Bureau of Standards, Gaithersburg, MD 20899

Aiken, R., Ed. (1985), Stiff Computation, Oxford Univ. Press, England Arakawa, A., and Lamb, V.R. (1977), "Computational design of the basic dynamical processes of the UCLA general circulation model," in General Circulation Models of the Atmosphere, J. Chang, ed., Methods in Computational Physics 17, Academic Press, N.Y., p.173

Atkinson, K.E. (1978), An Introduction to Numerical Analysis, J. Wiley \& Sons, New York 
Bagnaro, M., Laouisset, M., and Lockwood, F.C. (1981), "Modelisation d'un Incendie Simulé," in First Specialists' Meeting (International) of the Combustion Institute, Bordeaux (France), p.614

Bagnaro, M., Laouisset, M., and Lockwood, F.C. (1983), in J.G. Quintiere et al (eds) Fire Dynamics and Heat Transfer, ASME, NY, HTD-Vo1 25, p.107

Batchelor, G.K. (1967), An Introduction to Fluid Dynamics, Cambridge University Press, London, England

Baum, H.R. (1976), "An Analysis of Turbulent Mixing Layers Using a TwoEquation Model of Turbulence;" Proceedings Fron Second U.S./Japan Goverment Cooperative Program on Natural Resources (UJNR), unpublished

Baum, H.R. (1976), "An Analysis of Turbulent Mixing Layers Using a TwoEquation Model of Turbulence;" from second UJNR meeting.

Baum, H.R., Rehm, R.G., and Mulholland, G.W. (1982), "Computation of fireinduced flow and smoke coagulation," 19th Symposium (International) on Combustion, The Combustion Institute, Pittsburgh, PA, P.921

Baum, H.R., and Rehm, R.G. (1982), "Numerical computation of large-scale fireinduced flows," presented at the Eighth International Conf. on Numerical Methods in Fluid Dynamics, Aachen (Germany)

Baum, H.R., Rehm, R.G., Barnett, P.D., and Corley, D.M. (1983), "Finite difference calculations of buoyant convection in an enclosure, I. The basic algorithm," SIAM J. Sci. Stat. Comput. 4, p.117

Baum, H.R., and Rehm, R.G. (1984), "Finite difference solutions for internal waves in enclosures," SIAM J. Sci. Stat. Comput. 5, p.958

Baum, H.R., and Rehm, R.G. (1984a), "Calculations of three dimensional buoyant plumes in enclosures," Comb. Sci. and Tech. 40, p.55

Berl, W.G. (1961), editor, (International Symposium on) The Use of Models in Fire Research; National Academy of Sciences, Washington, D.C.

Birkhoff, G. (1983), "Numerical Fluid Dynamics," SIAM Review 25, p.1

Blomqvist, J. and Andersson, B. (1984), "Modelling of Furniture Experiments With Zone Models," Fire and Materials

Boisvert, R.F., and Sweet, R.A. (1984), "Mathematical software for elliptic boundary value problems;" chap. 9 in Cowel1 (1984)

Chung, G., Siu, N., and Apostolakis, G. (1984), "COMPBRN II: Code Description and Simulation of Experiments", UCLA-ENG-8404

Cooper, L.Y., and Forney, G.P. (1987), "Fire in a room with a hole: a prototype application of the Consolidated Compartment Fire Model (CCFM) computer code," Proceedings of the Eastern Section of the Combustion Institute; NBS, Gaithersburg, MD 20899, P.104

Corliss, G., and Chang, Y.F. (1982), "Solving Ordinary Differential Equations Using Taylor Series," ACM Transactions on Mathematical Software 8, No.2, P. 114

Cowe11, W.R. (editor) (1984), Sources and Development of Mathematical Software, Prentice-Hall, Inc., Englewood Cliffs, NJ

DiNenno, P., Beyler, C.L., Custer, R.L.P., Walton, W.D., and Watts. J.M., Jr., editors (1988); SFPE Handbook of Fire Protection Engineering; published jointly by the National Fire Protection Association, Quincy, MA, and the Society of Fire Protection Engineers, Boston, MA

Drummond, J.P. (1989), "Modeling of Supersonic Reacting Flowfields," this volume

Drysdale, D. (1985), An Introduction to Fire Dynamics, John Wiley \& Sons, N.Y. Duff, I.S. (1984), "A survey of sparse matrix software;" chap. 8 in Cowel1, 1984 Duong, D.Q. (1990), "The Accuracy of Computer Fire Models: Some Comparisons With Experimental Data From Australia," submitted to Fire Safety Journal 
Emmons, H.W. (1978), "A Note on Minimizing the Unknowns for Computation of a Large System of Equations," Home Fire Project Technical Report No. 26; Division of Applied Sciences, Harvard University, Cambridge, MA

Emmons, H.W. (1979), "The Prediction of Fires in Buildings," $17^{\text {th }}$ Symposium (Int'1) on Combustion, The Combustion Institute, Pittsburgh PA, p.1101 Fernandez-Pello, A.C. (1984), "Flame spread modeling," Combustion Science and Technology 39, p.119

Forsythe, G., and Moler, C.B. (1967), Computer Solutions of Linear Algebraic Systems, Prentice-Hall, Englewood Cliffs, N.J.

Fusegi, T., and Farouk, B. (1988), "Numerical Study on Interactions of Turbulent Convection and Radiation in Compartment Fires," To appear in Fire Science and Technology, an International Journal

Gahm, J.B. (1983), "Computer Fire Code VI - Volume 1," TR 58, Harvard University and NBS-GCR 83-451, National Bureau of Standards, Gaithersburg, MD

Galea, E.R. (1987), and Markatos, N.C., "Mathematical Modeling of Aircraft Cabin Fires," Appl. Math. Modeling 11, p.162

Galea, E.R. (1989), "On the Field Modeling Approach to the Simulation of Enclosure Fires," J. of Fire Protection Engineering 1, p.11

Gear, C.W. (1971), Simultaneous Numerical Solution of Differential-Algebraic Equations," IEEE Circuit Theory Transactions, 18, No.1 (Jan. 1971), p.89

Gosman, A.D., Humphrey, J.A.C., and Vlachos, N.S., "TEACH 3E; A General Computer Program for Three-Dimensional Recirculating Flow," Imperial College, Mech. Eng. Dept. Report.

Gross, D. and Robertson, A.F. (1965), "Experimental Fires in Enclosures," in Tenth Symposium (International) on Combustion, the Combustion Institute, Pittsburgh, PA, p.931

Gross, D., and Davis, W.D. (1988) "Burning Characteristics of Combat Ship Compartments and Vertical Fire Spread," NISTIR 88-3897, National Institute of Standards and Technology (U.S.), Gaithersburg, MD, Dec.88

Handa, T., Kawagoe, K., Yoshikawa, T., Mashige, J., and Joh, T. (1984), "Numerical simulation of early stage of a compartment fire," Fire Science and Tech. 4, P.91

Harlow, F.H., and Amsden, A.A. (1971), "Fluid dynamics, a LASL monograph," Report LA4700, Los Alamos Scientific Lab, Los Alamos, NM

Henrici, P. (1964), Elements of Numerical Analysis, J. Wiley \& Sons, N.Y. Hottel, H.C. and Sarofim, A.F. (1967), Radiative Transfer, McGraw-Hill, N.Y. Hutchings, B., and Iannuzzelli, R. (1987), "Taking the measure of fluid dynamics software," Mechanical Engineering, 109, May 1987, p.72

Hutchings, B., and Iannuzzelli, R. (1987a), "Benchmark problems for fluid dynamics codes," Mechanical Engineering, Vol. 109, June 1987, p.54

Ingberg, S.H. (1927), "Fire Tests of Office Occupancies," Q. NFPA 20, P. 243

Ingberg, S.H. (1928), "Tests of the severity of building fires," Q. NFPA 21 , P. 43

Jacobs, D. (Editor) (1978), Numerical Software - Needs and Availability; Academic Press, London/New York

Jarrat, P. (1970), in Numerical Hethods for Nonlinear Algebraic Equations, P. Rabinowitz, Ed., Gordon and Breach, N.Y.

Jeng, S-M., Chen, L-D., and Faeth, G.M (1982), "An investigation of axisymmetric buoyant turbulent diffusion flames," 19 th (International) Symposium on Combustion; The Combustion Institute, Pittsburgh, PA, p.349

Jones, W.P., and Launder, B.E. (1972), "The prediction of laminarization with a two-equation model of turbulence," Int. J. Heat Mass Transfer 15, 301

Jones, W.W. (1985), "A Multicompartment Model for the Spread of Fire, Smoke, and Toxic Gases", Fire Safety Journal 9, No. 1-2, p.55 
Kahaner, D.K., Moler, C., and Nash, S. (1989) Numerical Methods and Software, Prentice-Hall, Englewood Cliffs, NJ

Kawagoe, K. (1958), "Fire Behavior in Rooms," BRI Report No.27, Building Research Institute, Tokyo

Kreiss, H., and Oliger, J. (1973), "Methods for the approximate solution of time-dependent problems," GARP Publication Series 10, Global Atmospheric Research Programme

Ku, A.C., Doria, M.L., and Lloyd, J.R. (1977), "Numerical Modeling of Unsteady Buoyant Flows Generated by Fire in a Corridor, "13th (Int'l) Symposium on Combustion; The Combustion Institute, Pittsburgh, PA, p.1373

Kumar, S., and Cox, G. (1985), "Mathematical Modeling of Fires in Road Tunnels," 5th Int. Conf. on the Aerodynamics and Ventilation of Vehicular Tunnels, Lille; p.61

Kumar, S., Hoffman, N., and Cox, G. (1986), "Some Validation of JASMINE for Fires in Hospital Wards," in Numerical Simulations of Fluid Flow and Heat/Mass Transfer Processes, Eds. N.C. Markatos et al, Springer-Verlag, Berlin, p.159

Larson, D.W., and Viskanta, R. (1986), "Transient Combined Laminar Free Convection and Radiation in a Rectangular Enclosure, " J. Fluid Mech. 78, 65

Launder, B.E., and Spalding, D.B. (1972), Mathematical Models of Turbulence, Academic Press, London

Lawson, J.R., and Quintiere, J.G. (1985), "Slide-Rule Estimates of Fire Growth," NBSIR 85-3196, Nat'1 Bureau of Standards, Gaithersburg, MD

Levenberg, K. (1944) "A Method for the Solution of Certain Nonlinear Problems in Least Squares, Quart. Appl. Math. 2, p.164

Lewis, J., and Rehm, R.G. (1980), "The numerical solution of a nonseparable elliptic partial differential equation by preconditioned conjugate gradients," NBS J. Res. 85, p.367

Lloyd, J.R., Yang, K.T., and Liu, V.K. (1979), Proc. First Nat'1. Conf. on Numer. Methods in Heat Transfer (U. of Maryland, College Park, MD), 142

Lockwood, F.C., and Shah, N.G.(1976), "An Improved Model for the Calculation of Radiation Heat Transfer in Combustion Chambers," Proceedings ASMEAIChE, National Heat Transfer Conference, St. Louis

Lyons, J.W., Becker Jr, W.E., Clayton Jr, J.W., Emmons, H.W., Fristrom, R.M., Glassman, I., Graham, D.L., McDonald, D.W., and Nadeau, H.G. (1980), "Fire Research on Cellular Plastics: The Final Report of the Products Research Committee," PRC, Washington, D.C.

Lyons, J.W. (1985) Fire, Scientific American Library

Markatos, N.C., and Cox, G. (1981), "A Field Model for Smoke Flow in Enclosures," in First Specialists" Meeting (International) of the Combustion Institute, Bordeaux (France), p.653

Markatos, N.C., Malin, M.R., and Cox, G. (1982), "Mathematical Modeling of Buoyancy-Induced Smoke Flow in Enclosures," International J. Heat and Mass Transfer 25, p.63

Markatos, N.C. and Pericleous (1983), in J.G. Quintiere et al (eds) Fire Dynamics and Heat Transfer, ASME, NY, HTD-Vol 25, p.115

Markatos, N.C., and Cox, G. (1984), "Hydrodynamics and Heat Transfer in Enclosures," Physico.-Chem. Hydrody. 5 (No.1), p.53

Marquardt, D.W. (1963) "An Algorithm for Least-Squares Estimation of Nonlinear Parameters," J. Soc. Indust. App1. Math., 11, p.431

McCaffrey, B.J., and Dagher, S.N. (1989), "The Use of FIRST for Forced Counterflow-Ventilated Fires," preprint

Mitler, H.E. (1978), "The Physical Basis for the Harvard Computer Fire Code," Home Fire Project Technical Report No.34; Harvard University 
Mitler, H.E. and Emmons, H.W. (1981), "Documentation for CFC V, the Fifth Harvard Computer Fire Code", Home Fire Project Technical Report No.45, Harvard University, and National Bureau of Standards GCR 81-344

Mitler, H.E., and Rockett, J.A. (1981), "How Accurate is Mathematical Fire Modeling?" Presented at US-USSR Joint Seminar on Mathematical Modeling of Fire, Tbilisi, USSR, July 1981. Has also appeared as a publication of the Nat. Bur. of Standards (U.S.), NBSIR 86-3459 (1986)

Mitler, H.E. (1985), "The Harvard Fire Model", Fire Safety J. 9, No.1-2, p.7 Mitler, H.E. (1985a), "Comparison of Several Compartment Fire Models: An Interim Report," NBSIR 85-3233, National Bureau of Standards, Gaithersburg, MD 20899

Mitler, H.E., and Rockett, J.A. (1987), "Users' Guide to FIRST, a Comprehensive Single-Room Fire Model", NBSIR 87-3595; National Bureau of Standards, Gaithersburg, MD, 20899

Mitler, H.E. (1988), "Modeling Ignition," Section 5 in The Effect of Cigarette Characteristics on the Ignition of Soft Furnishings, by Gann, Harris, Krasny, Levine, Mitler, and Ohlemiller, NBS Technical Note 1241, National Bureau of Standards, Gaithersburg, MD 20899

Modak, A.T. (1978/9), "Radiation From Products of Combustion," Fire Research 1, P.339

Morton, B.R., Taylor, G.I., and Turner, J.S. (1956), "Turbulent Gravitational Convection From Maintained and Instantaneous Sources," Proc. Royal Society (London) A234, P.I

Nash, J.C. (1979), Compact Numerical Methods for Computers, J. Wiley, N.Y.

Nelson, H.E. (1986), "FIREFORM - A Computerized Collection of Convenient Fire Safety Calculations," NBSIR 86-3308; U.S. National Bureau of Standards, Gaithersburg, MD, 20899

Odeen, K. (1963), "Theoretical Study of Fire Characteristics in Enclosed Spaces," Bulletin 10, Div. of Building Construction, Royal Inst. of Technology, Stockholm, Sweden

Ohlemiller, T. (1985), "Modeling of smoldering combustion propagation," Progress in Energy Combustion Science 11, p.277

Oran, E., Young, T., and Boris, J. (1979), "Application of Time-Dependent Numerical Methods to the Description of Reactive Shocks," in $17^{\text {th }}$ (International) Symp. on Combustion; The Combustion Institute, Pittsburgh, PA, P.43

Parikh, J.S., Beyreis, J.R., and Christian, W.J. (1983), "Survey of the State of the Art of Mathematical Fire Modeling", Underwriters Laboratories, Northbrok, Illinois, UL Report File NC 554

Patankar, S.V., and Spalding, D.B. (1972) "A Calculation Procedure for Heat, Mass, and Momentum Transfer in 3-D Parabolic Flow, "Int. J. of Heat, Mass Transfer 15, 1987

Patankar, S.V. (1974), "A Computer Model for Three Dimensional Flow in Furnaces," Fourteenth International Symposium on Combustion; the Combustion Institute, Pittsburgh, PA., p.605

Patankar, S.V. (1980), Numerical Heat Transfer and Fluid Flow, Hemisphere Press, Washington D.C.

Peyret, R., and Taylor, T.D. (1983), Computational Methods frr Fluid Flow, Springer-Verlag, New York

Porsching, T.A. (1977), "A finite-difference method for thermally expandable fluid transients," Nuclear Science and Engineering 64, p.177

Quintiere, J.G. (1977), "The Growth of Fire in Building Compartments," ASTM Special Publication No. 614 
Quintiere, J.G. (1981), "An Approach to Modeling Wall Fire Spread in a Room", Fire Safety J. 3, p.201

Radhakrishnan, K. (1990), "Combustion Kinetics and Sensitivity Analysis Computations," this volume

Raithby, G.D., and Torrance, K.E. (1974), "Upstream-weighted Differencing Schemes and their Application to Elliptic Problems Involving Fluid Flow," Computers and Fluids 2, p.191

Ralston, A., A First Course in Numerical Analysis, McGraw-Hill, N.Y., 1965

Ramsde11, J.D. (1981), "Finding Minimal Feedback Vertex Sets," Home Fire Project Technical Report No.47; Harvard University, Cambridge, MA

Ramsde11, J.D. (1981), "Juggle User's Guide," Division of Applied Sciences, Harvard University, Cambridge, MA

Rehm, R.G., and Baum, H.R. (1978), "The equations of motion for thermally driven, buoyant flows," NBS J. Res. 83, p.297

Rehm, R.G., Baum, H.R., and Barnett, P.D. (1982), "Buoyant convection computed in a vorticity, stream-function formulation," NBS J. of Res. 87, p. 165

Rehm, R.G., Baum, H.R., Barnett, P.D., and Corley, D.M. (1985), "Finite difference calculations of buoyant convection in an enclosure, Part II: Verification of the nonlinear algorithm," Applied Numer. Math. 1, p.515

Reid, J.K. (1978), "Software for sparse matrices;" sec. II.3 in Jacobs (1978) Richtmyer, R.D., and Morton, K.W. (1967), Difference Methods for Initial-Value Problems, Second Ed., Interscience, New York

Roache, P.J. (1976), Computational Fluid Dynamics, Hermosa Publishers, NM

Rockett, J.A. (1982), "Modeling of NBS Mattress Tests With the Harvard Mark V Fire Simulator," NBSIR 81-2440, National Bureau of Standards, Gaithersburg, MD

Rockett, J.A., Morita, M., and Handa, T. (1983), "Using the Harvard fire simulation," Fire Science and Technology 3, No.1, p.57

Rockett, J.A. (1984), "Park Service room fire test simulations using the Harvard level 5.2 Computer Fire Model," NBSIR 83-2805, National Bureau of Standards, Gaithersburg, MD, 20899

Rockett, J.A., and Morita, M. (1985), "The NBS/Harvard VI Multi-Room Fire Simulation," Fire Science and Technology 5, No.2, p.159

Rockett, J.A., Morita, M., and Cooper, L.Y. (1989), "Comparison of NBS/Harvard VI Simulations and Full-Scale Multiroom Test Data," Fire Safety Journal 15, p. 115

Rodi, W., and Spalding, D.B. (1970), Wärme- und Stoffübertragung 3, p.85

Rotherme1, R.C. (1972), "A Mathematical Model for Predicting Fire Spread in Wildland Fuels," U. S. Department of Agriculture Forest Service Research Paper INT-115

Saffman, P.G. (1970), "A model of inhomogeneous turbulent flow, " Proc. Royal Soc. (London) A317, P.417

Satoh, K., Yang, K.T., and Lloyd, J.R. (1980), "Ventilation and Smoke Layer Thickness Through a Doorway of a Cubic Enclosure With a Central Volumetric Heat Source," Eastern Section of the Combustion Institute

Satoh, K., Lloyd, J.R., and Yang, K.T. (1984), "Flow and temperature oscillation of the fire in a cubic enclosure with ceiling and floor vents; III. Non-dimensional relationship between oscillatory frequency and heat source strength," Fire Research Inst. of Japan Report No. 57

Scarborough, J.B. (1962), Numerical Mathematical Analysis, 5th ed., Johns Hopkins Press, Laurel, MD

Schmidt, L.A., "A parametric study of probabilistic fire spread effects," Institute for Defence Analysis paper No. IDA P-1372, Sept. 1979 
Shampine, L.F., and Watts, H.A. (1984), "Software for ordinary differential equations;" chap. 6 in Cowel1 (1984)

Sibulkin, M. (1988), "Free convection diffusion flames from burning solid fuels," Prog. Energy Combust. Science 14, P.195

Simms, D.L., Hird, D., and Wright, H.G.H. (1960), "The Temperature and Duration of Fires, Part I: Some Experiments With Models With Restricted Ventilation," Fire Research Note \#412, Fire Research Station, Boreham Wood, England

Siu, N.O., "Probabilistic Models for the Behavior of Compartment Fires," Univ. of California at Los Angeles; NUREG/CR-2269 (1981)

Siu, N.O. (1982), "Physical Models for Compartment Fires", Reliability Engineering 3, 229

Smith, G.D. (1965) Numerical Solution of Partial Differential Equations, Oxford U. Press, N.Y.

Spalding, D.B. (1977) "The Calculation of Free Convection Phenomena in GasLiquid Mixtures," in Heat Transfer and Buoyant Convection, Eds. Spalding and Afgan, Hemisphere Pub. Co., Washington, DC

Steckler, K.D., Quintiere, J.G., and Rinkinen, W.J. (1982), "Flow Induced by Fire in a Compartment," 19 th Symposium (International) on Combustion; the Combustion Institute, Pittsburgh, PA, P.913

Stone, H.L. (1968), "Iterative solution of implicit approximation of multidimensional partial differential equations," SIAM J. Numer. Analysis 5, p. 530

Tanaka, T. (1983), "A Model of Multiroom Fire Spread," NBSIR 83-2718, National Bureau of Standards, Gaithersburg, MD 20899, and Fire Science and Technology 3, p.105

Thomas, P.H. (1960), "Studies of fires in buildings using models. Part 1 Experiments in ignition and fires in rooms, "Research Applied in Industry 13, p. 69

Thomas, P.H., and Hinkley, P.L. (1964), "Design of Roof Venting Systems for Single-Storey Buildings," Fire Research Tech. Paper No.10, Her Majesty's Stationary Office, London

Thomas, P.H., Heselden, A.J.M., and Law, M.B. (1967), "Fully-Developed Compartment Fires -. Two Kinds of Behaviox," Fire Research Tech. Paper No.16, London, H. M. Stationary Office

Todd, N.W., and Ryan, J.D. (1989), "Better Codes Via Fire Technology," Proceedings of the Pacific Rim Conference of Building Officials; International Conference of Building Officials, April 1989; p.293

Torrance, K.E. (1968), "Comparison of Finite-difference Computations of Natural Convection," NBS Journal of Research 72B, No.4, D.281

Torrance, K.E., and Rockett, J.A. (1969), "Numerical Study of Natural Convection in an Enclosure With Localized Heating From Below," J. Fluid Mech. 36, P. 33

Walsh, J. (1978), "Problems of algorithm design for partial differential equations;" Section V.4 in Jacobs (1978)

Watts, H.A., and Shampine, L.F. (1979), "DEPAC - Design of a User-Oriented Package of ODE Solvers," SAND-79-2374, Sandia Labs., Albuquerque, NM

Williamson, R.B. (1976), "Fire Performance Under Full Scale Test Conditions -. A State Transition model," in 16th (International) Symposium on Combus. tion; The Combustion Institute, Pittsburgh, PA, p.1357

Yang, H.Q. (1987), "Laminar Buoyant Flow Transition in Three-Dimensional Rectangular Enclosures," Ph.D. Thesis, Univ. of Notre Dame, Notre Dame, Indiana 
Yang, K.T., Lloyd, J.R., Kanury, A.M., and Satoh, K. (1984), "Modeling of Turbulent Buoyant Flows in Aircraft Cabins," Comb. Sci. and Tech. 39, P. 107

Young, T.R., and Boris, J.P. (1977), "A Numerical Technique for Solving Stiff Ordinary Differential Equations Associated With the Chemical Kinetics of Reactive-Flow Problems," J. of Phys. Chem. 81, p.2424

Young, T.R. (1980), "CHEMEQ - A Subroutine for Solving Stiff Ordinary Differential Equations," NRL Memorandum Report 4091, Naval Research Laboratory, Washington, D.C. 


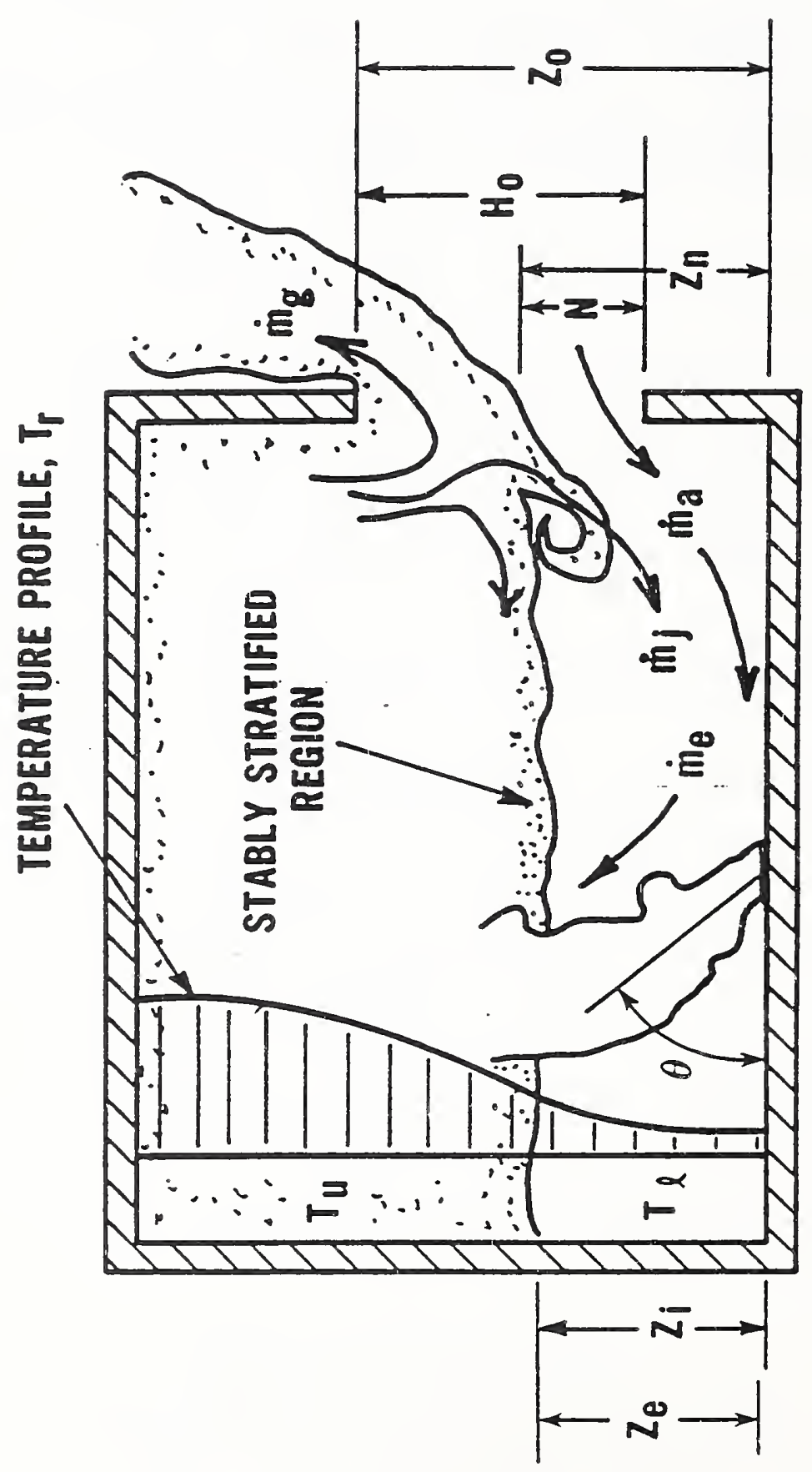

-

记

ญ

霡

भ 0

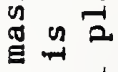

ข 듕

언

ชี ญ

$>\rightarrow$

远

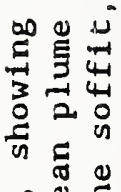

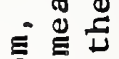

:

स

范

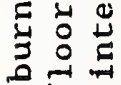

这

4 ب

0

$\perp_{0}^{-1} \frac{1}{0}$

T.

总出 气ِ

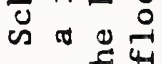

入

-

됭 क

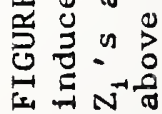




\begin{tabular}{|c|c|c|c|}
\hline \multirow{3}{*}{\multicolumn{2}{|c|}{$\begin{array}{r}\text { U.S. DEPARTMENT OF COMMERCE } \\
\text { NATIONAL INSTITUTE OF STANDARDS AND TECHNOLOGY }\end{array}$}} & \multicolumn{2}{|c|}{$\begin{array}{l}\text { 1. PUBLCATION OR REPOAT NUMBER } \\
\text { NISTIR } 90-4294\end{array}$} \\
\hline & & \multicolumn{2}{|c|}{$\begin{array}{l}\text { 2. PERFORMIMO ORGAMIZATION REPOAT MUMBER } \\
\text { Issued MaY } 1991\end{array}$} \\
\hline & & \multicolumn{2}{|c|}{ 3. PUBUCATION DATE } \\
\hline \multicolumn{4}{|c|}{$\begin{array}{l}\text { 4. TITLEAND SUBTITL } \\
\text { Mathematical Modeling of Enclosure Fires }\end{array}$} \\
\hline \multicolumn{4}{|c|}{$\begin{array}{l}\text { 5. AUTHOA(S) } \\
\text { Henri E. Mitler }\end{array}$} \\
\hline 6. $\begin{array}{ll}P \\
u \\
\text { a }\end{array}$ & $\begin{array}{l}\text { U.S. DEPARTMENT OF COMNERCE } \\
\text { MATIONAL INSTTMUTE OF STANDARDS AND TECHMOLOQT } \\
\text { GATHERSBUAG, MD } 2089\end{array}$ & \multicolumn{2}{|c|}{ 7. CONTRACT/ORANT MUMBER } \\
\hline \multicolumn{4}{|c|}{ 9. SPOMSORING ORCANIZATION MAME AND COMPLETE AODRESS (STRETT, CTT, STATE, ZM) } \\
\hline \multicolumn{4}{|c|}{ 10. SUPPLEMEMTAAY MOTES } \\
\hline \multicolumn{4}{|c|}{ 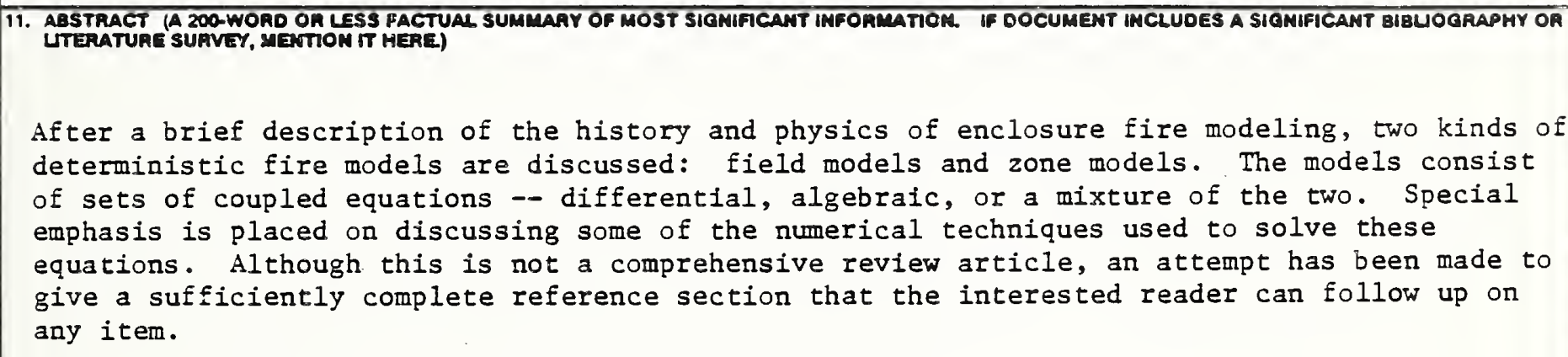 } \\
\hline \multicolumn{4}{|c|}{$\begin{array}{l}\text { 12. KEY WOADS (6 TO } 12 \text { ENTRIES; ALPHABETICAL OADER; CAPTTALIE ONLY PROPER MAMES; AND SEPARATE KEY WORDS IY SEMICOLONS) } \\
\text { computer models; fire models; mathematical models; modeling; numerical methods; room fires }\end{array}$} \\
\hline \multirow{3}{*}{\multicolumn{3}{|c|}{ 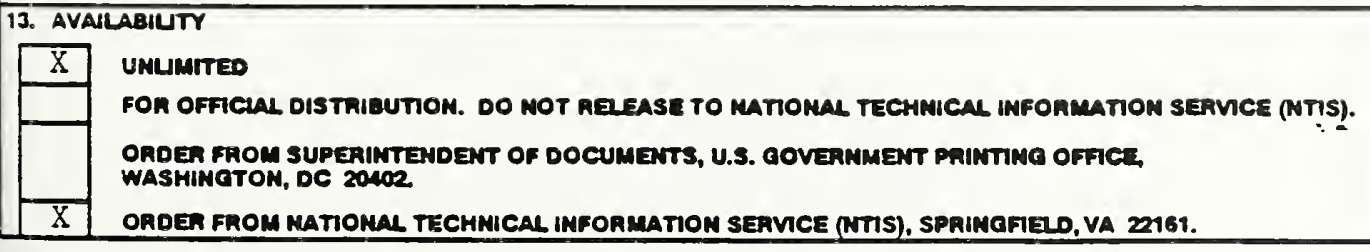 }} & 14. NUMBEA OF PRINTED PAGES \\
\hline & & & 47 \\
\hline & & & $\begin{array}{r}\text { 15. PAICE } \\
\text { A03 }\end{array}$ \\
\hline
\end{tabular}





\section{.}




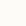

